Abstract

Recently, there has been an increased interest in the topic of "wireless ATM." The subject matter raises interest due to the potential combination of two of the hottest topics in communications of the mid-'90s, but at the same time raises a natural question as to its viability, and sometimes even its desirability. In this article the authors survey potential applications of wireless ATM and describe what is usually meant by wireless

ATM, why it may make sense, and some of the proposals to build systems based on it. The emphasis in this article is on the physical layer, the data link layer, and the access layer. The authors also present general observations on each of these layers, and some research solutions to these problems. Mobility issues and interoperability with the existing networks are addressed. Finally,

the authors list some of the proposals to build wireless ATM systems from the literature.

\title{
Wireless ATM: Limits, Challenges, and Proposals
}

\author{
ENDER Ayanoglu, Kai Y. ENg, AND MARK J. KAROL
}

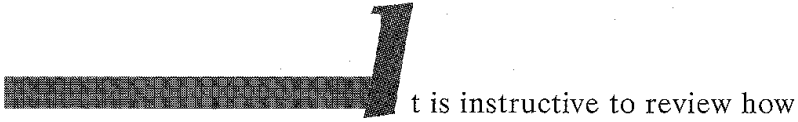
asynchronous transfer mode (ATM) technology came into being in order to discuss why wireless ATM makes any sense or not. ATM technology is the result of efforts to devise a transmission and networking technology to provide high-speed integrated services: a single infrastructure for data, voice, and video. It evolved from the wide area into the local area, and then into the access loop. The following subsections describe how this evolution took place.
\end{abstract}

\section{ATM in the Wide Area Network}

Integrated services digital network (ISDN), which was proposed for integrated communications, took a very long time to standardize, and when the standardization was completed it received a lukewarm reception from network and access providers on the grounds that the market for it did not exist. ${ }^{1}$ Technically, there are two problems with ISDN: its mainly circuit-switched nature and the rates selected. The circuitswitched nature of ISDN makes it difficult to provide bandwidth on demand, which is a requirement for data and compressed video. Also, with the standardized transmission rates, basic rate interface (BRI) and primary rate interface (PRI) $(128 \mathrm{~kb} / \mathrm{s}$ and $1.5 \mathrm{Mb} / \mathrm{s})$, ISDN is insufficient to provide broadcast-quality video. This lack of serious networking capability for ISDN resulted in a standardization effort for an essentially packet-switched service with higher transmission rates. After long debates in the communications engineering community, in the late ' 80 s a proposal, known as asynchronous time division (ATD), first outlined why fixed packets should

\footnotetext{
1 Only when conventional modems reached their fundamental limits in terms of transmission speeds did the local exchange carriers begin offering the service in limited ways. This is partially due to the fact that ISDN equipment for the local exchange network became widely available only then, and also due to the fact that they did not see a market for ISDN in the local exchange loop until more widespread use of computers at home became commonplace. As will be described in a subsection in the sequel, there are several candidate technologies for providing $A T M$ to the home via the local exchange loop, the cable TV plant, or new fiber, and with what these can offer, ISDN as a long-term networking enabler does not seem viable other than as a transitional technology. On the other hand, due to the uncertainties in customer demands, when the wide-scale deployment of these services will take place is unclear; and hence this tran sition may take some time.
}

be the norm. The size of the packets was based on considerations of voice transmission at $64 \mathrm{~kb} / \mathrm{s}$, and it described a header pattern for the fixed-size packets [1]. In a very short time, this proposal, renamed ATM, was under serious study at the International Telecommunications Union - Telecommunications Standards Sector (ITU-T) (then the CCITT), the international standardization body of the telecommunications industry. The ITU-T standardization activity followed some medium characteristics and some performance benchmarks; the typical bit error rate assumed was about $10^{-10}$, and the typical ATM packet (cell) loss rate was designed to be about $10^{-6}$.

\section{Local ATM}

In the beginning of the ' $90 \mathrm{~s}$, the computer industry began studying ways to replace its low-speed $(10 \mathrm{Mb} / \mathrm{s})$ networking infrastructure, which consisted primarily of Ethernet and token ring networks. A local area network (LAN) standard at about $100 \mathrm{Mb} / \mathrm{s}$, fiber distributed data interface (FDDI), was agreed on and development efforts began, but FDDI was a data networking standard, not an integrated communications standard. In particular, it had no simple means to integrate circuit-switched traffic onto the network. A bandwidth reservation technique for circuit-switched data in FDDI, FDDI-II, was proposed, but did not catch on. The LAN community turned to the standardization activity going on in the metropolitan area network (MAN) and wide area network (WAN) communities for ATM, and with the appeal of the simple translation of packets destined for MANs and WANs in the LANs, decided to adopt ATM as a LAN technology to provide multimedia [2]. Soon after this effort, the LAN industry took the initiative in ATM standardization, and a de facto standardization organization, the ATM Forum, was founded. As of this writing, the ATM Forum is the organization where ATM standardization, especially in terms of the local area, is taking place. The ATM Forum makes decisions based on a majority vote of the participating companies, and therefore can move much faster than ITU-T, which requires consensus among its participants, who have the status of national representatives. Although the immediate interest in ATM to the desktop has recently somewhat slowed down with the availability of inexpensive $100-\mathrm{Mb} / \mathrm{s}$ switched Ethernets, the potential to integrate data and real-time services with quality of service (QOS) guarantees still makes ATM the ultimate networking alternative on the desktop, and in the LAN, MAN, and WAN. 


\section{ATM to the Home}

A third area where ATM is a candidate is the residential video delivery community. With the improvement in hybrid fiber coax (HFC), fiber to the curb (FTTC), fiber to the home (FTTH), and asymmetric, broadband, or very-high-speed digital subscriber loop (ADSL, BDSL, or VDSL) technologies, both cable TV operators and local exchange carriers are becoming interested in residential video delivery. It should be emphasized that the delivery of video to residential customers generates opportunities for providing interactive services, and with the explosion of the World Wide Web and related Internet services, use of the local exchange loop, coaxial cable TV, and fiber are potential ways to bring video and interactive services to the home. The unifying transmission mode for all of these service alternatives is ATM. The ATM Forum has recently established a residential broadband subnetworking group to specify standard interfaces for such applications [3].

\section{The Role of Wireless in ATM}

Wireless ATM is mainly considered as an "access to an ATM network" issue. Depending on what kind of ATM network is to be accessed, different aspects of wireless networking need to be addressed. In the LAN scenario, wireless ATM as an extension of the LAN for mobile users, or for simplifying wiring or reconfiguration, has appeal. In the ATM-to-the-home scenario, wireless ATM has the appeal of extending the set-top box into portable appliances such as television receivers. More important, it has the potential to address a need to provide residential access for alternate carriers after deregulation of the local communication markets, which is happening very fast in various parts of the world.

\section{The Need for Wireless ATM}

A typical reaction to the concept of wireless ATM is to question the compatibility of several aspects of the ATM protocol and the wireless channel. First, considering the fact that ATM was designed for media whose bit error rates are very low (about $10^{-10}$ ), it is questioned whether ATM will work at all in the highly noisy wireless environment. The environment in question is typically a multiaccess channel that may be also time-varying. Second, the wireless channel is an expensive resource in terms of bandwidth, whereas ATM was designed for bandwidth-rich environments. ATM effectively trades off bandwidth for simplicity in switching. Every ATM cell carries a header with an overhead of about 10 percent. Even this much overhead is considered too high for the shared radio channel. In addition, the potential need to transmit single ATM cells means the system should be capable of transmitting individual cells. However, the physical layer overhead associated with the transmission of individual cells, due to channel equalization and timing (synchronization), can exceed this 10 percent overhead and potentially reach the size of an ATM cell, or even exceed it. If so, then the inefficiency of the resulting system may outweigh the advantages of wireless access.

However, if these problems are solved, there are significant advantages to wireless ATM. For example, there will be soft- ware that uses ATM as the transport medium. As a wireless LAN application, wireless ATM provides this software seamless access to an ATM network. This is beneficial for mobile users as well as for reconfigurability. Also, residential video delivery applications are useful for providing broadband access to residences without investing in new infrastructure.

\section{Native Mode or TCP/IP over ATM?}

$T^{\mathrm{t}}$

is generally considered that ATM networking has two potential modes to pursue. The first mode is commonly referred to as "native mode ATM" and means that application programs directly use ATM by means of an adaptation layer (Fig. 1). The second mode provides ATM as a transport medium for the existing Transmission Control Protocol/ Internet Protocol (TCP/IP) suite, and is known as "TCP/IP over ATM" (Fig. 2). It is expected that most video and voice applications will use native mode ATM, whereas data applications may use TCP/IP over ATM or native mode ATM. Accordingly, it can be expected that wireless residential video delivery applications will use native mode ATM, whereas for a wireless LAN both TCP/IP over ATM and native mode ATM will be applicable (Fig. 3).

There are significant differences between the support of native mode ATM or TCP/IP over ATM in terms of wireless ATM, especially for wireless LAN applications. In a wireless LAN, users may be mobile. In order to provide seamless communications under the mobility of users provisions have been made in TCP/IP, and various proposals known as Mobile IP exist. However, these provisions are mainly for data applications, and it is not clear that they can work satisfactorily for audio and video. There is room for providing mobility management using ATM, and proposals for doing that exist. We will elaborate on a mobility management technique using ATM cells in a later section.

Another issue regarding TCP/IP over ATM versus the native ATM solution is related to the radio. The question in this case is where the segmentation and reassembly for ATM will be made, or where the ATM adaptation layer will be located. If this location is the base station, requirements on the radio can be simplified because packet sizes can be larger (IP packets are large). The main disadvantage of this approach is the more complicated base station whose complexity increases with the average number of active users. Our main interest in this article is to provide an infrastructure for ATM cell transport over wireless channels, and therefore we will concentrate on the case in which the adaptation layer is located in the mobile.

\section{Physical-Layer Issues for Wireless Packet (ATM) Networks}

$T$ he physical layer deals with the actual transmission of data over the physical medium by means of a radio or an optical transmitter/receiver pair. At this time, radios that operate in a burst and multi-access mode at $20+\mathrm{Mb} / \mathrm{s}$ are not commercially available, but are the focus of many 


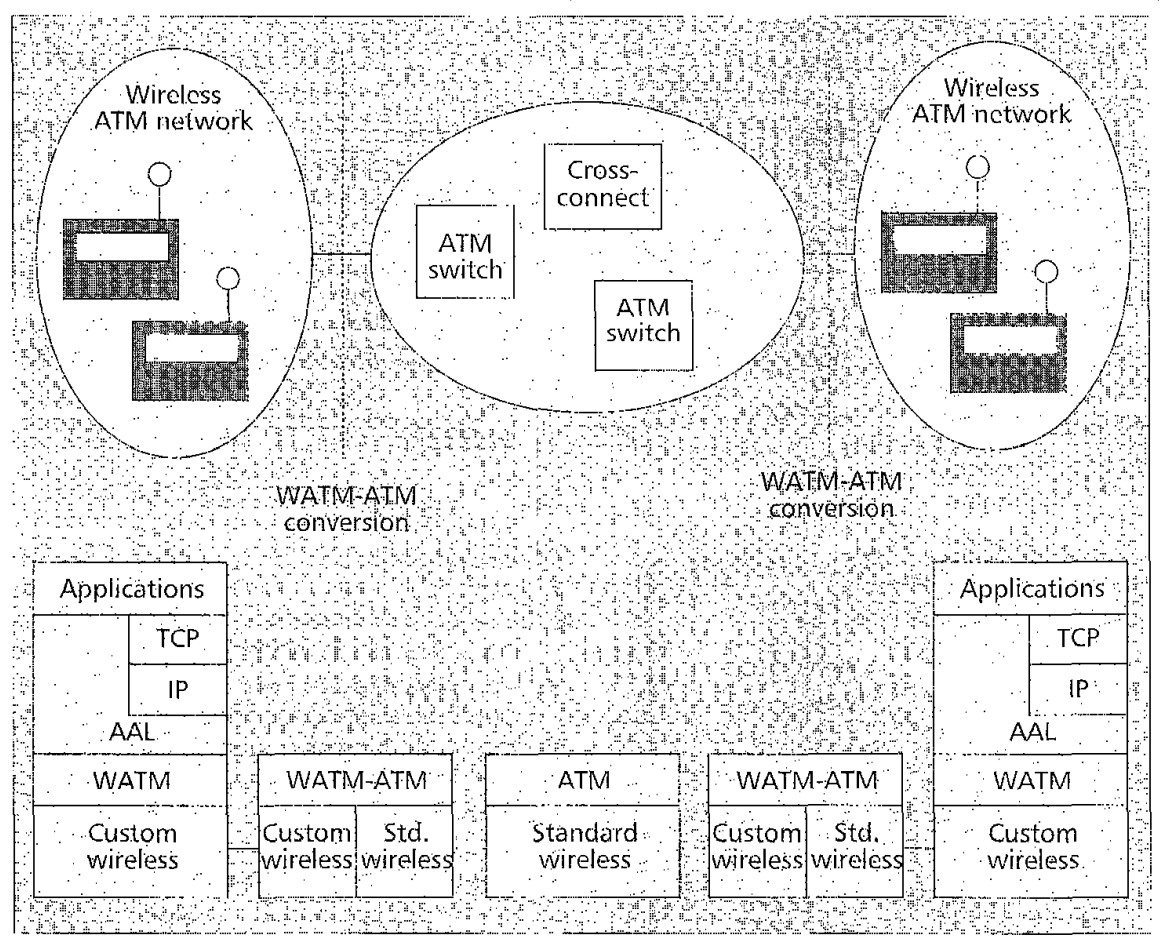

Figure 3. A wireless ATM system and the corresponding protocol stacks.

need to be solved, which takes additional time and reduces efficiency even further. There are various approaches in the communications engineering literature to address these issues; however, although various different attempts have been made, no single technique has yet emerged as the clear winner in addressing all problems. It is expected that the solution will be formulated by a combination of analysis, simulations, prototyping, and experimentation in the coming years. Details of some proposals will be elaborated on later.

\section{Infrared vs. Radio}

Infrared systems can be less expensive to build than radio systems. Infrared receivers detect the amplitude or position of optical signals, not the frequency or the phase, and therefore can be built by simple power detection. Therefore, a significant part of the cost of a radio due to frequency conversion or quadrature demodulation can

research projects. The main challenge in this endeavor is overcoming multipath reception from stationary and moving objects, which results in a space- and time-varying dispersive channel. Equalization techniques to solve this problem are well known, but they require training sequences to learn the channel. For a burst-mode modem, the time-varying channel needs to be learned often, potentially each time a packet is to be transmitted, especially if packets are coming from different sources. This generates an overhead, which results in a necessary reduction in the efficiency of the system. Keeping this overhead to its minimum is a research challenge that can be addressed by intelligent modulation and equalization techniques. There are additional problems, such as carrier recovery, that reduce the efficiency of the system, as well as techniques such as channel coding that can improve the coverage of the system. A detailed discussion of all these issues is not possible here. Instead, we provide a list of issues, together with main options, their fundamental limits, pertinent implementation issues, and conclusions reached with the available data in the following subsections.

The most important reason why building an efficient packet radio in the indoor environment is difficult is multipath effects. Multipath, due to reflections from stationary and moving objects, generates a channel that has intersymbol interference or, in the spectral domain, a nonflat transmission channel. Intersymbol interference becomes a significant problem when path lengths differ by a significant fraction of the symbol time. If they differ by a multiple of half a wavelength, the interfering paths can cancel each other, which is known as a fade, and the phenomenon is known as Rayleigh fading. For infrared, where the wavelengths are extremely small, Rayleigh fading is not an issue because of the extreme short distance of a fade. However, the intersymbol interference due to multipath can still be a problem. Established equalization techniques solve the intersymbol interference problem, but require training, which is time-consuming, reduces the efficiency of the system, and increases its cost.

For a burst modem, timing and carrier recovery problems be avoided. Furthermore, since infrared frequencies are not regulated, licensing is not an issue, which can help keep costs low. There are privacy advantages to keeping transmissions within a room with infrared, which also simplifies the system since all cells may then be identical.

By diffusing an infrared beam by means of a lens, a system similar to radio in terms of an indoor coverage area can be obtained. This solution suffers from technical problems, especially in terms of multipath and synchronization, similar to those of radio. In addition, such systems are more difficult to implement because of the difficulty of providing multiple access and in achieving a wide coverage area. These systems also have shorter range than radio of the same rate. They are preferable when electromagnetic interference is a concern, such as in hospitals, where electromagnetic interference with sensitive medical equipment cannot be risked.

By using direct (or aimed) infrared, multipath problems can be avoided; therefore, very high speeds are possible. Such a system requires line-of-sight transmission and a high degree of accuracy in pointing the infrared beam. The latter can be a problem in populated indoor and outdoor environments. The former, pointing the beam, is nontrivial, and is further complicated by the potential need for frequent realignment. Furthermore, the need for highly directed beams makes multi-access difficult. The operating area for such a system would be restricted to a spot, which takes away significant utility of a wireless LAN. For outdoor applications, extreme intensity of solar radiation constrains an infrared system by severely restricting its angle of reception.

In summary, for high-speed indoor or outdoor wireless packet networks, radio is the preferred solution because it is not restricted to line of sight and does not require pointing, and multi-access is simpler to attain. Infrared is an option for high-speed networks with pointed links that essentially operate as a collection of point-to-point communication links, or for solving electromagnetic interference problems. At low speeds, diffused wireless LANs restricted to a single room are possible. 


\section{Circuit-Switched vs. Packet-Switched Operation}

Circuit-switched transmission is attractive from the viewpoint of modem design. A circuit-switched system is one where the link is continuously active, data is always available, and timing and carrier recovery and channel equalization functions can be performed continuously, in the "data-directed" mode rather than in a separate training mode. Hence, this circuitswitched operation simplifies the significant operational problems present in burst modem operation. For circuit-switched data, or continuous bit rate operation, this alternative is a good solution. In an ATM scenario, it provides the option to carry out ATM cell segmentation and reassembly at either the mobile or the base station. Multiple access for this system requires a separate signaling channel dedicated to call setup and teardown. This solution is not well suited to individual cell mode transmission.

Packet switching is the natural operation mode for variable bit rate operation. The potential need to transmit single ATM cells requires large overhead due to equalization, and carrier and timing recovery. The design of the radio to accommodate the use of data from previous cell transmissions to reduce this overhead is possible, but complicates the design of the radio. The picture is further complicated by the need to allow for different qualities of service. Of course, by trading off bandwidth for complexity, all the problems mentioned above become much easier to tackle. Since the radio spectrum is a shared public resource, this trade-off is only possible by duplication of the system in space (i.e., by a microcellular network). Nevertheless, the radio frequency (RF) spectrum is too valuable to waste freely, and the system must be engineered very carefully.

Different switching techniques for real-time constant bit rate data and variable bit rate data may make sense for wireless, since this may simplify the problems mentioned above. For example, FDDI-II has such a hybrid system, but due to the difficulties of implementation it never caught on.

In summary, due to the presence of variable bit rate services, a pure circuit-switched system should be ruled out for wireless ATM. The most desirable system is a packet-switched one with QoS guarantees. The operational difficulties of providing any QoS makes a hybrid system a final alternative, by trading off a potentially more complicated implementation.

\section{Operating Frequency}

A basic limitation in terms of operating frequency is at about $10 \mathrm{GHz}$. Implementation beyond $10 \mathrm{GHz}$ is difficult with today's technology. Silicon elements do not operate at these frequencies. Gallium arsenide (GaAs) operates but is expensive. Another problem is propagation: due to the standard propagation formula, attenuation has the characteristics of 20 $\log f$ where $f$ is frequency, and at these frequencies the electromagnetic wave behaves similar to an optics wave. This restricts the range and, in an indoor environment, restricts transmission to inside the room.

For less than $10 \mathrm{GHz}$, Federal Communications Commission (FCC) allocations exist. The issue in that case is which band to use, and whether to operate in a licensed or an unlicensed band. We discuss this issue in the next subsection.

\section{Licensed vs. Unlicensed Bands}

In the United States, the FCC has allocated certain spectral bands for uses potentially open to applications of wireless ATM. Some of these bands are designated "unlicensed": the band can be used by certain equipment as long as it adheres to certain operational procedures. Two general sets of these bands are industrial, scientific, and medical (ISM) and personal communications services (PCS). The remainder of the bands are licensed; operation in those bands is contingent on licensing agreements with the FCC, which are difficult to obtain.

In 1985 , three ISM bands were opened by the FCC to communications applications with the condition that they use spread spectrum techniques or very low power. One of these bands is available worldwide, others only in North America (U.S. and Canada) [4]:

- $915 \mathrm{MHz}$ band (902-928 MHz, $26 \mathrm{MHz}$ bandwidth): This band, only available in North America, is highly crowded. and expected to get even more crowded. Many existing users are non-spread-spectrum applications, which is a problem for spread spectrum communications.

- $2.4 \mathrm{GHz}$ band (2.4-2.4835 GHz, 83.5 MHz bandwidth): This band is lightly loaded, but interference from microwave ovens is a problem.
For high-speed indoor or outdoor wireless tworks, radio is the preferred solution because it is not restricted to line of sight and does not require pointing, and multi-access is simpler to attain.

- $5.8 \mathrm{GHz}$ band (5.725-5.85 GHz, $125 \mathrm{MHz}$ bandwidth): This band is lightly loaded; some radar interference exists, but it has limited coverage ( 80 percent with directional antenna) and is only available in North America. Currently, operation in this band requires GaAs components, which are expensive:

The requirement of spread spectrum makes ISM bands undesirable for wireless ATM at rates of about $25 \mathrm{Mb} / \mathrm{s}$ since the needed chip rates are prohibitively high.

Also, the FCC opened a band at $1.9 \mathrm{GHz}$ for unlicensed PCS operations:

- $1.9 \mathrm{GHz}$ band (1910-1920 MHz, $10 \mathrm{MHz}$ bandwidth): The PCS band is 1850-1990 MHz. The part between 1910 and $1920 \mathrm{MHz}$ is allocated for unlicensed asynchronous or packet-switched applications, that between 1920 and 1930 $\mathrm{MHz}$ for unlicensed synchronous or circuit-switched applications; the rest of the PCS band is allocated for licensed applications [5]. There is a band open in Europe around 1.9 $\mathrm{GHz}$ for digital enhanced cellular telephone (DECT). In the United States, use of this band requires "etiquette" (listen before transmitting; if there are others, do not transmit). The band is currently occupied by other users with microwave point-to-point links, who will clear the band in five years. In this band, peer-to-peer devices are not allowed before nationwide clearance. There is not enough bandwidth available in this band to transmit $25 \mathrm{Mb} / \mathrm{s}$.

Recently, an industry organization known as WINForum requested of the FCC that a new band be opened. This band is:

- $5.2 \mathrm{GHz}$ band: This band is open in Europe with a bandwidth of $100-150 \mathrm{MHz}$ (depending on national regulations). WINForum requested of the FCC that it be opened in the United States with a $250 \mathrm{MHz}$ bandwidth, called SUPERNET. There is an additional petition by Apple that a $300 \mathrm{MHz}$ band be opened at the 5.2 and $5.8 \mathrm{GHz}$ bands $(5.15-5.3$ $\mathrm{GHz}$ and 5.725-5.875 $\mathrm{GHz}$ ) for community networks.

This band is the most promising in terms of wireless ATM 


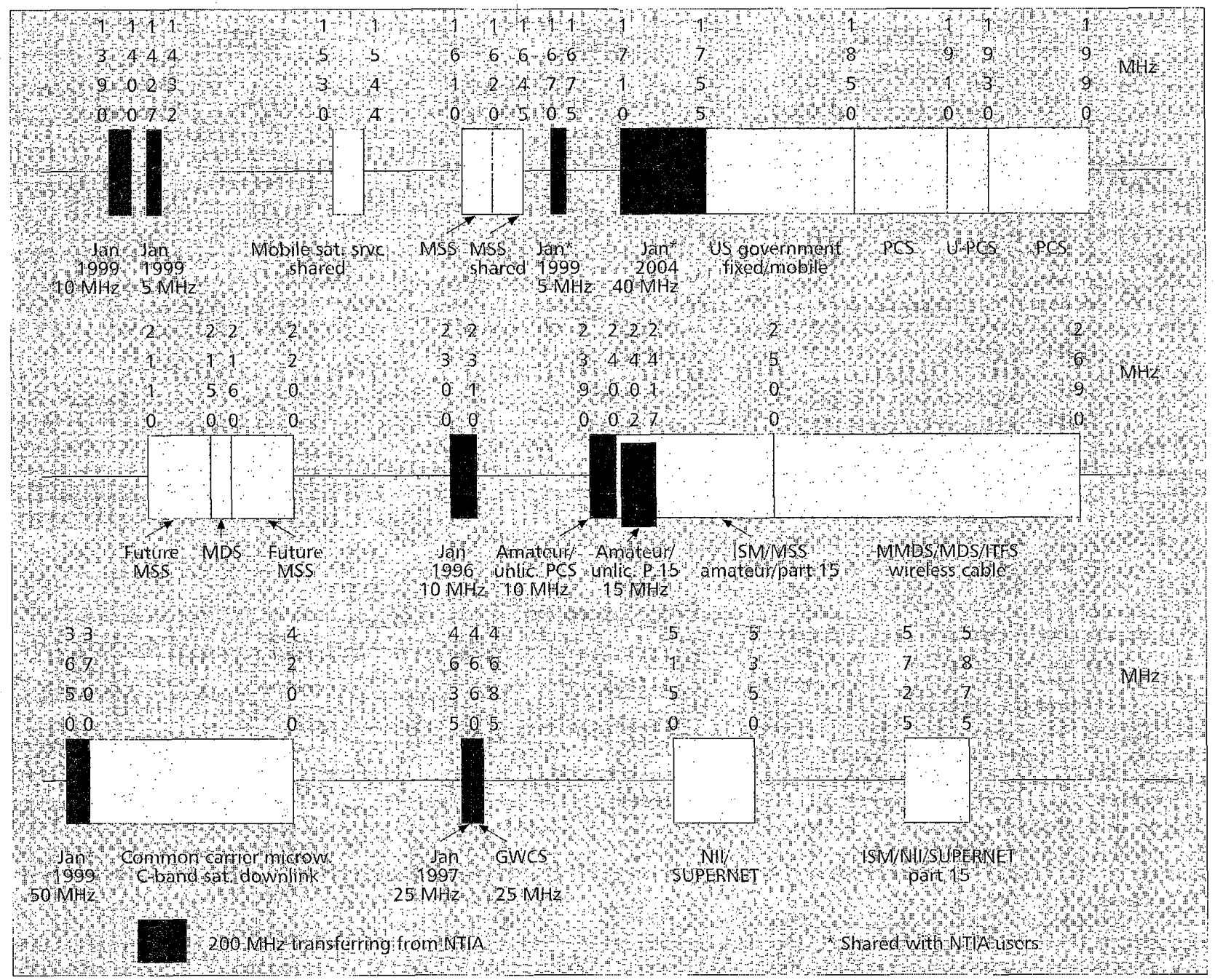

Figure 4. Spectrum allocation in the United States between 1 and 6 GHz. MSS: mobile satellite service, PCS: personal communication services, U-PCS: unlicensed PCS, MDS: multipoint distribution service, ISM: industrial, scientific, medical, MMDS: multichannel multipoint distribution service, ITFS: instructional television fixed service, GWCS: general wireless communication services, NII: national information infrastructure, NTLA: National Telecommunications and Information Administration.

applications. At the end of April 1996, the FCC announced plans to set aside the SUPERNET and national information infrastructure (NII) bands (5.15-5.35 and 5.725-5.875 GHz) for free and unlicensed high-speed communications over short distances. The plan is to allow free and unlicensed transmission using not more than $100 \mathrm{~mW}$ effective isotropic radiated power (EIRP) of power. At the same time, the FCC also asked for comment on the proposal by Apple, which would make it possible to transmit higher power levels.

Above $10 \mathrm{GHz}$, in Europe, the European Telecommunications Standards Institute (ETSI) has designated the 17.1-17.3 $\mathrm{GHz}$ band for wireless LAN operation.

The licensed bands require FCC approval, which is a long and difficult process. The performance will be a premium in licensed bands since the band is not shared with other users. As such, the licensed band operation is preferable for outdoor applications, in particular for residential broadband delivery.

There is more bandwidth becoming available for licensed and unlicensed operation in the 1-6 GHz band (Fig. 4) and above $6 \mathrm{GHz}$ (Fig. 5)

Technology for licensed or unlicensed operation exists. For experimental purposes, it makes sense to start with a system that operates in the unlicensed band and carry the design over to the licensed band for premium applications. The decision has significant impact on the proposed system. In terms of bandwidth and spectrum availability, a multimegabit-per-second system can be built in the $1.9 \mathrm{GHz}$ band with etiquette, or in the $5.2 \mathrm{GHz}$ band when it is opened. For high-volume premium applications, a licensed band $<10 \mathrm{GHz}$ should be investigated.

\section{Spread Spectrum}

Wireless systems based on spread spectrum techniques are being used increasingly for telephony, mainly because of higher capacity arguments in the literature. In the case of broadband wireless LAN applications, the capacity increase is secondary and other criteria become important. Two types of spread spectrum techniques exist: direct sequence and frequency hopping. For a $10-\mathrm{Mb} / \mathrm{s}$ system, with a direct sequence spread spectrum technique and processing gains of 10-100, chip rates of $100 \mathrm{Mb} / \mathrm{s}$ to $1 \mathrm{~Gb} / \mathrm{s}$ are needed, which are difficult to achieve, and the system is therefore expensive. In addition, the near-far problem requires power control, which results in added complexity. For a wireless LAN, frequency hopping is implementable, and it helps solve the multipath problem; however, frequency hopping is difficult for data applications because of synchronization problems. Although it 


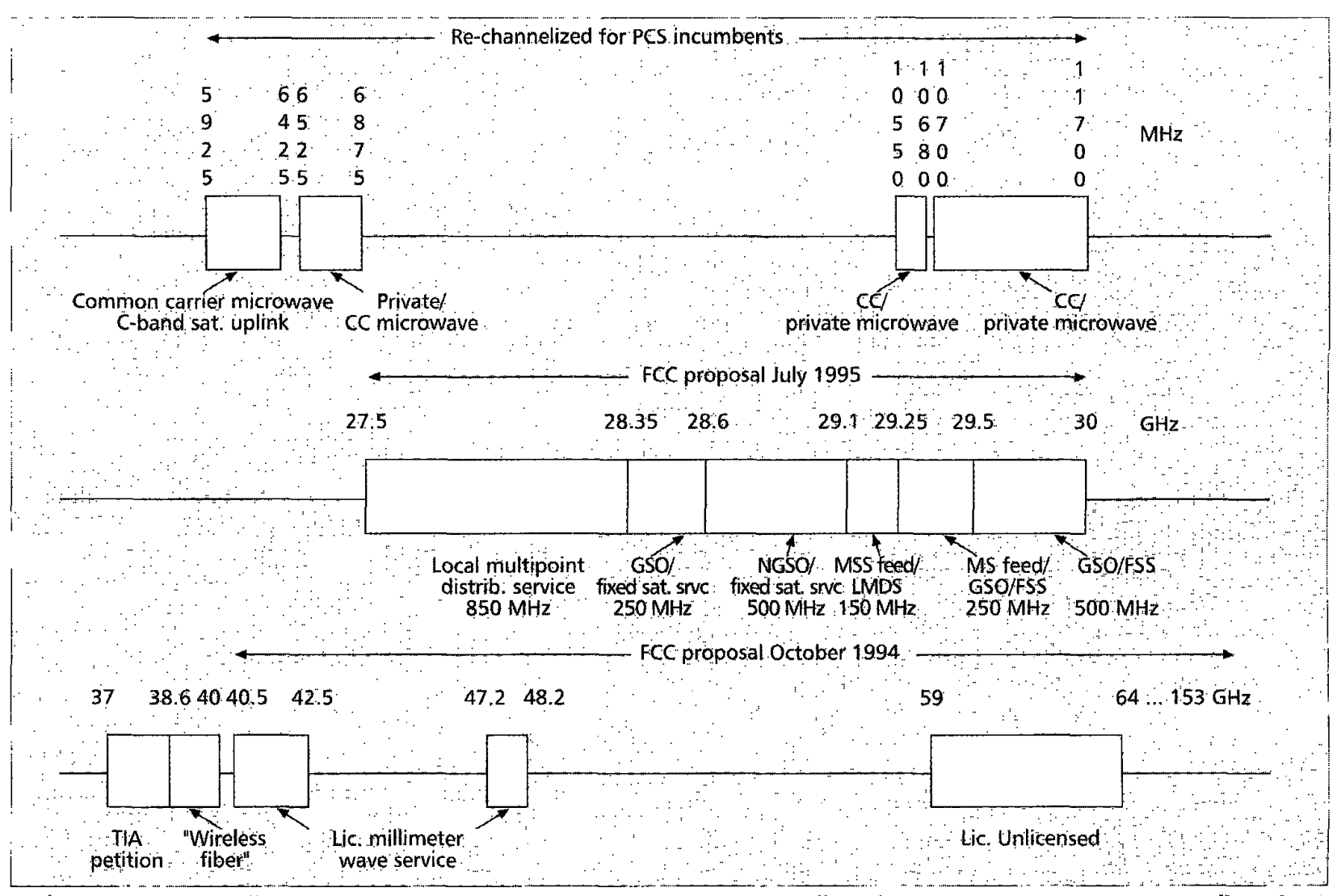

Tigure 5. Spectrum allocation in the U.S. above $6 \mathrm{GHz}$. GSO: geostationary satellite orbits, NGSO: non-geostationary satellite orbits, LMDS: local multipoint distribution service, FSS: fixed satellite service, TLA: Telecommunications Industry Association.

is true that there are currently several wireless LANs that employ spread spectrum techniques, they were designed to operate in the ISM bands, and therefore were required to use spread spectrum.

In summary, for a wireless LAN spread spectrum is difficult at high bit rates and not really needed. For low-bit-rate access applications, direct sequence spread spectrum may have a place due to potential capacity increase, and additionally for voice applications to provide soft handoffs. The capacity calculations in direct sequence spread spectrum are controversial, and depend on the basic assumptions. This subject is an open research topic. In general, it is not expected that high-speed wireless LANs will employ spread spectrum due to the difficulty of achieving high bit rates [6].

\section{Modulation}

Linear modulation techniques known as binary phase-shift keying (BPSK), quaternary PSK (QPSK), differential PSK (DPSK), and quadrature amplitude modulation (QAM) are possible for radio. Due to the high level of noise and the difficulty of equalization, multilevel constellations are difficult to achieve. The disadvantages of such a system are that a significant equalization effort is needed, and amplification is difficult. The amplification problem can be solved by constant amplitude modulation schemes, in particular by minimum shift keying (MSK) and GMSK. With constant amplitude waveforms, nonlinear amplifiers can be employed, which get around the difficulty of linear amplification. A very desirable property of MSK and Gaussian MSK (GMSK) is that although they have constant amplitude, they can be implemented using a quadrature-type architecture (similar to linear modulation techniques).

Another modulation scheme, known as orthogonal fre- quency division multiplexing (OFDM), has several desirable properties: it simplifies equalization considerably, and it has graceful performance degradation, and because of the absence of equalization, lower complexity [7]. A 150-Mb/s OFDM wireless network has been reported in the literature [8]. There are studies in the literature that show that under Rayleigh fading the delay and throughput performance of a multichannel approach outperforms that of a single-channel one [9]. Further studies in the literature show that with OFDM in an indoor environment, only 6 percent of measured channels at 5 $\mathrm{GHz}$ resulted in an error floor greater that $10^{-5}$, compared with 46 percent for single-carrier differential QPSK (DQPSK); at $17 \mathrm{GHz}$ the results are even more favorable [10]. More advanced techniques, such as trellis-coded modulation (TCM), can be useful in providing protection against noise; however, TCM at $10 \mathrm{Mb} / \mathrm{s}$ is very difficult to implement, especially under the bursty error characteristics of the wireless channel. ${ }^{2}$

In summary, a radio that operates at about $25 \mathrm{Mb} / \mathrm{s}$ using QPSK or GMSK is feasible. The OFDM alternative is attractive, but requires further research before wide-scale adoption. At this point, technologies based on TCM remain out of the question for bit rates as high as $10 \mathrm{Mb} / \mathrm{s}$.

\section{Equalization}

The least mean square (LMS) algorithm is the most commonly used equalization algorithm because of its simplicity and stability. Its implementation complexity is about $4 N$, where $N$ is the number of taps in the channel impulse response to be

${ }^{2}$ TCM systems for the fading channel need to be designed using set-partitioning criteria different than those for the additive white Gaussian noise (AWGN) channel [11]. 
A multirate system is one where high bit rates are employed at high signal-to-noise ratios; when the signal-to-noise ratio is low, the system is switched to a low bit rate. Such a system is not difficult to implement provided that a highly reliable feed-

equalized. Its main disadvantage is its relatively slow convergence. LMS converges in 100-1000 symbols, where a symbol in QPSK or DQPSK is equal to 2 bits. Thus, $100-1000$ symbols correspond to 25 to 250 bytes, which is from one-half to five ATM cells.

A faster equalization technique is known as recursive least squares (RLS). There exist various versions of RLS with somewhat different complexity (computation and coding) and convergence trade-offs [12]. The origins of RLS are in solving sets of linear equations. RLS, with a complexity of $17 \mathrm{~N}$ symbols, is more difficult to implement than LMS, but converges in fewer symbols. In indoor propagation simulations, about 60 symbols have been observed to be sufficient for RLS to converge. This convergence time depends on the number of taps, but not on the channel to a significant extent, whereas LMS depends on the eigenvalue spread of the channel. In addition to increased complexity, RLS has known stability problems. This makes LMS the first choice in implementing equalization. Although some manufacturers employ RLS in some GSM receivers, RLS for wideband wireless applications is still a research topic.

\section{Carrier and Timing Recovery}

Carrier and timing recovery add delay to processing, and therefore reduce the efficiency of a burst modem. Carrier recovery takes $5-10 \mu \mathrm{s}$ at 2.4 and $5.2 \mathrm{GHz}$. At $20 \mathrm{Mb} / \mathrm{s}$, this is equal to about $12-24$ bytes, which is about $25-50$ percent of an ATM cell. Differential techniques reduce the need for accurate carriers; differential GMSK is attractive and is an open research topic. Timing recovery is less significant than carrier recovery; it can be accomplished by two-pass algorithms at the expense of additional delay.

Carrier and timing recovery add overhead in a burst modem, less than needed for equalization, but still large. The reduction of this overhead is an open research topic.

\section{Channel Coding}

There is no consensus in the literature on the feasibility of channel coding for wireless channels. The main issue is the bursty nature of wireless channel errors. The bursts are very long, and typically break the interleaving depths used for conventional physical-layer coding systems. Large interleaving depths are more difficult to implement and increase the endto-end delay. The wireless channel, especially the indoor wireless channel, can be characterized as being bimodal: either it has no errors, or it has many errors. Under these circumstances, conventional physical-layer error correction will not help. However, system performance improves by incorporating error correction in unconventional ways. For example, by replacing the cyclic redundancy check (CRC) in the data link layer with an error detecting and correcting code, the advantages of error correction can be obtained by at most a slight increase in the CRC field size. Or by using cell-level forward error correction, significant performance gains for the situation described above can be obtained without interleaving delay [13]. Cell-level coding transmits redundant cells for protecting data cells that cannot be corrected by using physical layer or per-cell error correction. Thus, cell-level coding provides an additional layer of error protection for long fades. In addition, physical-layer coding has the potential to improve the range of the system, and may be a viable technique for the multirate system with a variable coding rate. back channel exists. Since the information rate to be transmitted on this channel is very low, such a channel can be obtained by redundant transmissions without much difficulty. This system provides a communication link at low signal-to-noise ratios, which is a highly desirable feature.

\section{Multiple Antennas}

Multiple antennas improve performance. A simple technique is to use multiple antennas and choose the one with the best signal-to-interference ratio. Even this simple algorithm provides a definite performance improvement. More complicated techniques, such as adaptive antenna arrays, will improve performance substantially [14]; however, they require too much complexity for megabit-per-second rates. Selective antennas with simple selection algorithms are plausible and provide a sufficiently large degree of improvement.

\section{Transmission Capacity}

Transmission capacity calculations are usually made using bandwidth efficiency figures of modulation techniques. Standard bandwidth efficiency figures for various communication systems are available; however, they are derived mostly for the additive white Gaussian noise (AWGN) channel. Currently, wireless multipath channel characteristics are finding their way into bandwidth efficiency calculations [15]. In general, a radio that can approach a bandwidth efficiency of $1-2 \mathrm{~b} / \mathrm{s} / \mathrm{Hz}$ with a single nondirectional antenna is considered feasible, while with multiple antennas this figure increases linearly with the number of antennas [15].

\section{Data Link Layer Issues for Wireless Packet (ATM) Networks}

\section{Encapsulation}

Encapsulation is a technique used for transporting the protocol data units (PDUs) of one protocol within those of another. In its simplest form, the PDUs of the former protocol are placed within the headers and trailers of the new protocol, which are stripped off at the end, where the latter protocol is terminated. The advantage of this technique is the transparency it provides; the disadvantages are the added overhead and encapsulation and decapsulation delays. Encapsulation and decapsulation delays can be minimized by using cut-through techniques (switch right after reading the header), and the added overhead can be minimized by reducing the unnecessary overhead to a minimum (e.g., by using header compression).

\section{Header Compression}

One of the issues in wireless ATM is the 10 percent header overhead inherent in ATM. This overhead, if present in every ATM cell, causes a large degree of unnecessary inefficiency. In the media for which ATM is designed, bandwidth is abundant, and the 10 percent overhead is not an issue. In the wireless medium, however, this is not tolerable. On the other hand, most of the time the header a mobile host utilizes is fixed or one of a few alternatives. Thus, the information content of a header can easily be represented with a smaller number of bits than the 40 bits used in conventional ATM. There are several obvious possibilities for header compression; we will describe one below in the example protocol. 


\section{$A R Q$ vs. FEC}

Automatic repeat request (ARQ) and forward error correction (FEC) techniques have been compared for various channels, in particular the wireless channel, by many authors. The issue is quite complicated for integrated communications in a wireless channel, due to various conflicting requirements. Finding an engineering solution between ARQ and FEC that will reduce complexity and minimize extra bandwidth and delay, while at the same time satisfying all QoS requirements, is difficult. There are three main ARQ techniques: stop-and-wait, continuous ARQ or go-back- $N$, and selective repeat or reject. ARQ with selective repeat is the most complicated of the three, but it is time-efficient, provided a reverse channel always exists and the forward channel can be re-accessed immediately whenever the repeat request is received. In a multi-access system these latter requirements cannot always be accommodated. There are techniques that reserve slots for the transmission of acknowledgment (ACK) messages and the repeated packets, but this uses extra bandwidth for reliability and is similar to FEC in spirit. Another possibility is to treat ACKs and retransmitted packets with high priority, but this does not guarantee QoS all the time and complicates the otherwise simple ARQ method. FEC is considered useful when packet transmission time becomes smaller with respect to the transmission delay, and when there is extra bandwidth to burn. It is simple in terms of time management and does not require keeping copies of transmitted packets, a reverse channel, or the immediate accessibility of the reverse channel. It has operational complexity, but that can be handled in hardware. The main disadvantage of FEC is the constant burning of extra bandwidth even when there are no errors. On the other hand, simulations indicate that errors in a wireless channel are highly correlated, and an adaptive technique has the potential to provide a variable overhead.

The best technique is a combination of ARQ and FEC. Although several hybrid ARQ/FEC techniques have been reported in the literature, particular characteristics of the wireless channel and availability of the reverse and forward channels under the multi-access protocol change many of the boundary conditions of the problem, and new research results under these conditions are needed.

In general, data applications that are not delay-sensitive can be transmitted with ARQ, while real-time applications such as video and audio are better transmitted with FEC.

\section{Quality-of-Service Issues}

As stated above, it is difficult to satisfy QoS requirements for delay-sensitive applications by using pure $A R Q$. In the absence of channel errors, QoS issues can be taken care of by the multi-access layer. When channel errors are present, however, the multi-access layer has to work in conjunction with the data link layer to satisfy varying requirements. Satisfaction of all possible QoS requirements under wireless channel noise in a multi-access system is a tall order. A more realistic proposal is to divide the $\mathrm{OoS}$ requirements into a few classes, with zero probability of loss at one end and as small delay as possible at the other. The former is served best with ARQ, the latter with FEC.

\section{Split Functionality}

For a cellular outdoor network similar to the wireless phone network, the mobile has limited batterry power and usually limited central processing unit (CPU) power. On the other hand, the base station does not have battery power limitations, and can potentially have more CPU power than the mobile. Under these circumstances, a data link layer protocol designed to move the complexity of the operations to the base can provide CPU cycle savings. Such a protocol is described in [16]. For a wireless LAN, similar ideas may or may not hold, depending on the complexity allowed at the base. For example, the base stations in [17] are designed with simplicity in mind; therefore, the base has more limited CPU power. On the other hand, the mobile has limited battery power; therefore, the asymmetry is to the advantage of the base in terms of battery power and to the advantage of the mobile in terms of processing complexity. Therefore, a split-functionality data link layer is not appropriate for this application. On the other hand, for residential broadband delivery applications, the asymmetry can potentially be applied so that the residential unit has most of the complexity, since neither the residential unit nor the base runs on batteries, and it is desirable to reduce the load on the base.

\section{An Example Protocol}

This is an example of a reliable data link layer protocol to transport ATM cells ( 48 bytes payload +5 bytes header) over a wireless point-to-point link. The wireless link can have a large number of errors, whereas ATM is designed for very reliable media such as optical fiber. The protocol ensures that the cells are transported reliably by combining a sliding window transport mechanism with selective repeat ARQ and FEC. It minimizes ATM header overhead over the air by means of header compression. The protocol provides per-cell FEC whose size can be changed adaptively. It also provides parity cells for recovery from errors that cannot be corrected using the per-cell FEC field. The number of these cells, as well as the size of a window (frame), can also be adaptively changed. In addition, the window can be terminated prematurely to request an immediate ACK message and to satisfy QoS requirements. The sliding window with selective repeat mechanism may require a large buffer due to the need to deliver cells in sequence. With today's operating systems delivering buffer space on request, this limitation is easily and efficiently overcome. The following describes the most basic elements of the protocol; additional messages for enhancing the operation can be added without altering the basic operation described below, and without altering the main contribution.

The protocol transfers messages in a window (frame) (Fig. 6). The window consists of three kinds of distinct messages: control, information, and parity. The size of each message will be clear from the bits at the beginning of each message, so the recipient can determine where to go to process the next message.

There are three types of control messages. Each control message begins with a field ( 1 bit) equal to 0 , identifying it as a control message. 


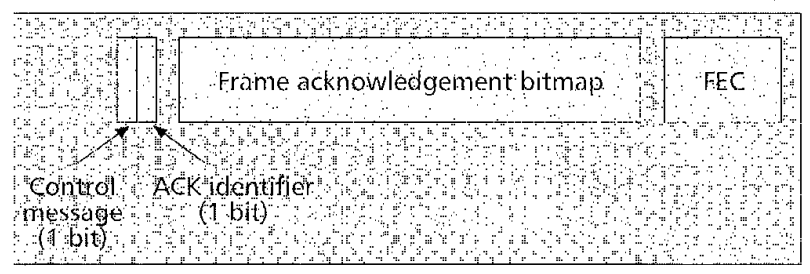

- Figure 7. An ACK message. The size of the bitmap is determined based on the number of I-cells in the frame, and is known to both the transmitter and the receiver.

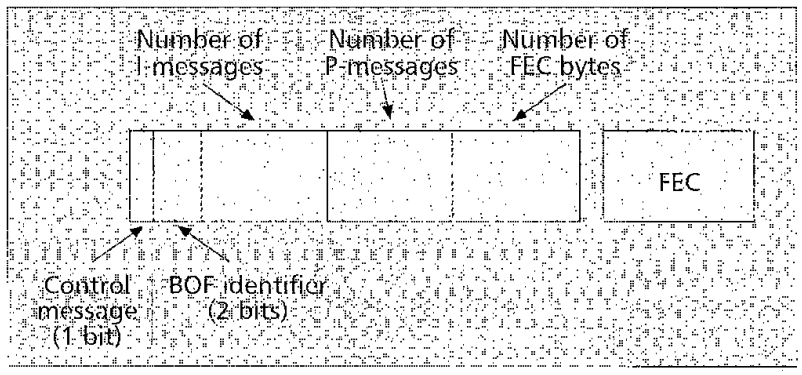

Figure 8. $A$ BOF message. The sizes of the last three fields are small, based on the incremental size desciptor explained in the text.

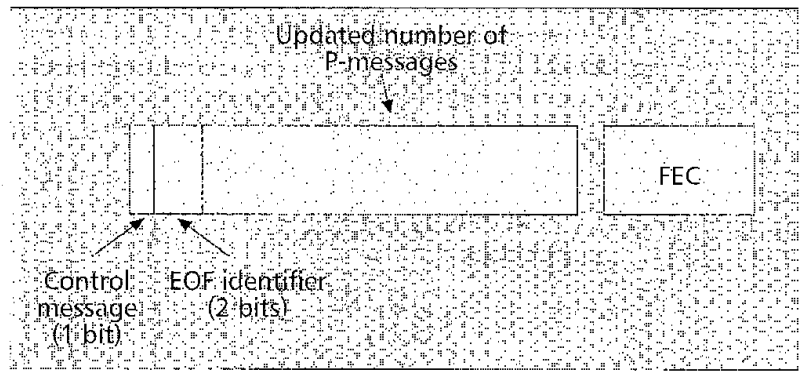

Figure 9. An EOF message. The size of the last field is larger than for the BOF message, since in this case the incremental descriptor is not used.

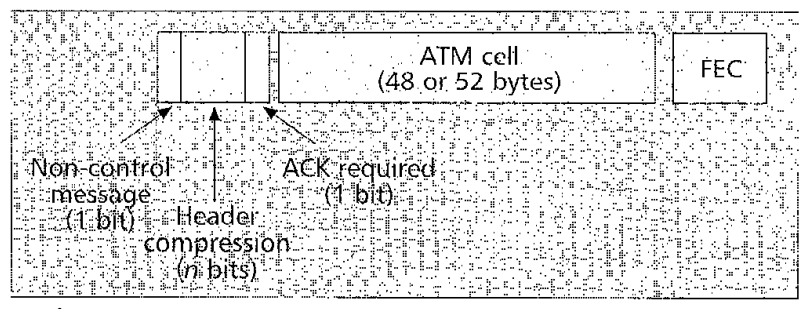

Figure 10. An information message (I in Fig. 6). Depending on the header compression field, the ATM cell is 48 (only payload) or 52 bytes (full cell minus HEC).

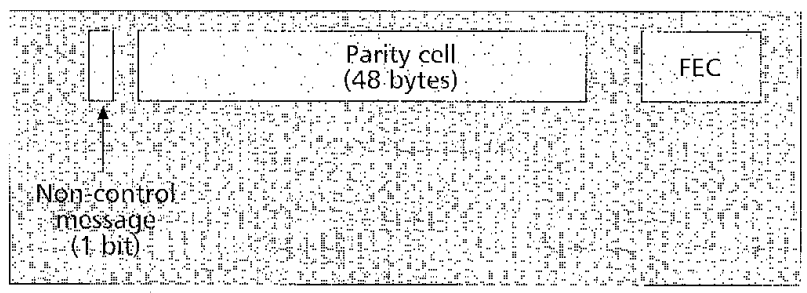

Figure 11. A parity message ( $P$ in Fig. 6 ). The size of the parity message, calculated based on the payloads of the I messages in the frame, is 48 bytes. The 1-bit field for indicating a noncontrol message serves the purpose of being able to introduce ACK messages between $P$ messages, and is optional.
The first type of control message is a block ACK transmitted from the receiver of a transmission to the transmitter (Fig. 7). The second bit in the message is set to 0, thus identified as this ACK message. The size of the message depends on the size of the window, and is known by both the transmitter and the receiver, based on prior information exchange. The ACK message itself consists of a bit map that has a 1 for each information cell (ATM cell) correctly received, and a 0 for each ATM cell not correctly received within the window. An ATM cell that was in fact received in error, but whose errors are corrected by using the per-cell FEC or by means of the parity cells, is considered an ATM cell that is correctly received for the purposes of the ACK message. An ACK message is appended by a FEC field.

The second type of control message, beginning of frame (BOF), is transmitted at the beginning of each frame (Fig. 8). The second bit of this message is set to 1 and the third bit to 0 . This message has three additional fields: the first indicates the number of information cells in the frame, the second indicates the number of parity cells in the frame, and the third indicates the number of bytes used in the per-cell FEC. In order to minimize the overhead for these fields, the information is transmitted incrementally. For example, two bits are used to identify the following four cases:

- The default value

- Same as the previous value

- Increment the previous value

- Decrement the previous value.

More bits may be used to accommodate several default values and several increment/decrement values. For example, with three bits, three default values, two increment and decrement values each, and the no-change message can be accommodated (other variations are possible). BOF employs a FEC field whose size is always constant or is given by the previous BOF.

The third kind of control message indicates the end of frame (EOF) when one wishes to terminate a frame prematurely (to override the value provided by BOF) (Fig. 9). The second bit of this message is set to 0 , and the third bit is set to 1 . This message indicates the number of parity cells to be appended since that number may change due to the termination of the frame. EOF employs an FEC field whose size is given by $\mathrm{BOF}$.

Each information cell is prepended by a short field (Fig. $10)$. Depending on this field, an information cell is a complete ATM cell (minus the HEC field) or only the payload of the ATM cell. This field has the following subfields. The first bit is equal to 1 , identifying what follows as a noncontrol message. The second field is used to compress the header overhead. In its simplest form, this field consists of a single bit. For one value of this bit (e.g., 1), the virtual path identifier/virtual circuit identifier (VPI/VCI) field of this cell is equal to that of the previous cell, and therefore the header is not transmitted; for the other value (e.g., 0) the VPI/VCI field is not equal to that of the previous cell, and therefore the full ATM cell (minus the HEC field) is transmitted. More generally, $n$ bits are used in this field. There are $2^{n-1}$ registers that hold VPI/VCI information. The first bit in the field is a set or read command. When this bit is used to set a register, the VPI/VCI field of the ATM cell is stored in the register, and the full ATM cell (minus the HEC field) is transmitted. When this field is used to read a register, the addressed register's VPI/VCI field is recalled from the memory at the receiver, and therefore only the payload of the ATM cell is transmitted over the air. For example, 0000 may mean to set register 0 with the VPI/VCI value of the existing cell, 1000 may mean to read this register, 0001 may mean to 
set register 1 with the VPI/VCI of the existing cell, and 1001 may mean to read register 1 . In this example, there are 8 registers, 0 through 7 . Whenever a set register value is used, the full ATM cell (minus the HEC field) is transported. After an ATM cell is transported and its VPI/VCI identified either by transmission or through the memory, the appropriate HEC field is added at the receiver. Another bit in the field is used by the transmitter to state that an ACK on this cell is not expected from the receiver (used, e.g., for real-time messages).

After the last information cell or the EOF cell, parity cells are placed in the frame (Fig. 11). Parity cells are constructed using the payloads of information cells; therefore, their size is equal to 48 bytes each. Parity cells need not be prepended by the beginning of cell fields for parsing purposes; however, in order to be able to insert an ACK message between parity cells, a field to identify them as noncontrol messages can be used as an option. Parity cells are not acknowledged.

\section{MPEG-2 Video Transport Employing FEC in an Indoor Channel}

A study of the performance of error control based on FEC for Motion Picture Experts Group version 2 (MPEG-2) video transmission in an indoor wireless ATM LAN was carried out in $[13,18]$ where combined source and channel coding techniques that employ single-layer and scalable MPEG-2 coding to combat channel errors were compared. In this study, an indoor time division multiple access (TDMA) scenario with the following parameters was considered: mobile velocity (corresponding to people walking in an office) is $5 \mathrm{mph}$, bit rate is $4.5 \mathrm{Mb} / \mathrm{s}$, delay spread between two faded paths of $88 \mathrm{~ns}$ which corresponds to a mid-size office, with a packet size of 54 bytes ( 48 byte ATM cell payload +6 byte compressed header, sequence number, and a per-cell error control field). Figure 12 shows the trace of a typical error event for this case using Jakes' model [19]. The top figure shows a broad range, while the next figure focuses on one of the bursts, showing their duration (about five packets) and magnitude. There are a few bursts of packets in error, and in each burst the number of bytes in error per packet is large. Note that when most of the bytes in the packet are in error, interleaving within the

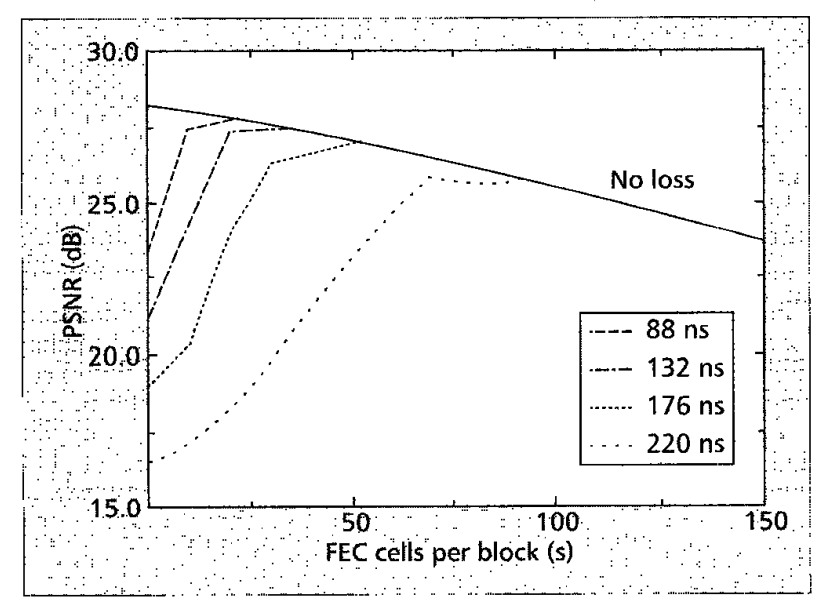

Figure 13. Effect of cell-level coding as a function of delay spread. cell will not help. As soon as we determine that a packet is in error, we know that the channel is bad and the next few packets are likely to be in error. This suggests that per-window, redundant-packet-based FEC can be beneficial in correcting these errors.

When FEC is employed without any ARQ, error control in the wireless link is achieved by means of a channel encoderdecoder (codec). In this system, Reed-Solomon encoding/decoding is chosen because it leads to minimal overhead bandwidth. For byte-level FEC, the Reed-Solomon (RS) encoder processes symbols where a symbol is a group of $m$ $=8$ bits. The R-S encoder processes $N$ data symbols and generates an overhead of $2 t$ symbols. The value $t$ is the number of errored symbols that can be corrected by the R-S coder. With sequence numbers in cells, it is possible to detect the location of lost cells. If the error locations are known, $k$ R-S symbols are sufficient to recover from $k$ errored symbols. Then, to encode blocks of cells, up to $s$ lost cells can be recovered by means of $s$ redundant cells using an R-S coder. As described above, this method of FEC is called cell-level coding.

It is well known that encoded video is extremely sensitive to the temporal and spatial location at which a cell loss occurs. To decrease the sensitivity of the results to the location of cell losses, each simulation in $[13,18]$ was run 10 times using a different random number seed for the cell loss process for each run. The results presented in $[13,18]$ are the average value over all runs for each simulation. The results for a video sequence were evaluated using the peak signal-to-noise ratio (PSNR) as an objective measure of video quality. Although PSNR results do not correspond directly to subjective evaluation, in general, a reduction in PSNR from the lossless channel performance begins to be visually noticeable for values greater than $0.5 \mathrm{~dB}$ and is clearly visible for values greater than $1 \mathrm{~dB}$.

Several video coding techniques were investigated. The single-layer video coder used in the simulations is an MPEG-2 main profile encoder operating at an output bit rate of 4 $\mathrm{Mb} / \mathrm{s}$. Performance of two different MPEG-2-based two-layer video coding techniques were also investigated. The first, data partitioning (DP), is based on dividing the encoded discrete cosine transform (DCT) coefficients into two groups: low-frequency components in a base layer which is given high priority (HP), and high-frequency components into an enhancement layer which is given low priority (LP). The second, SNR scalability (SNRS), first uses a coarse quantizer to encode DCT coefficients to create a base layer given HP, and then uses a fine quantizer on the errors to create an enhancement layer 


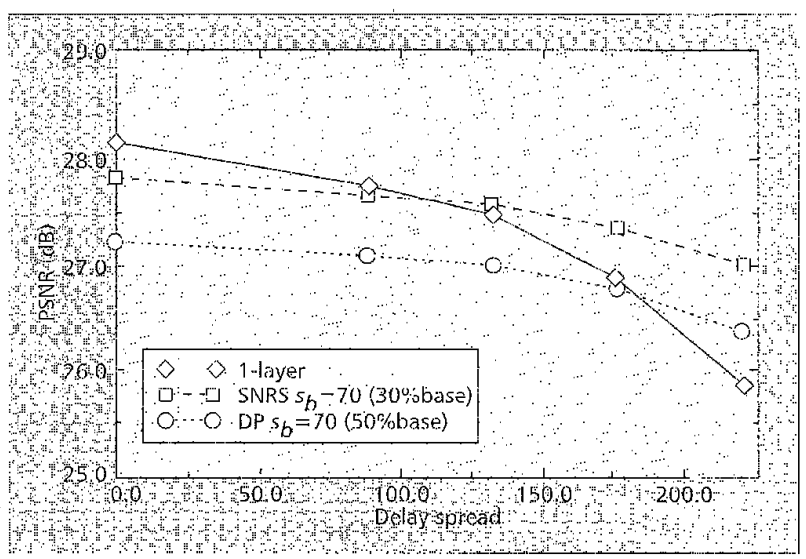

Eigure 14. Optimal quality for 1- and 1-layer MPEG-2 in an indoor environment.

given LP. In terms of implementation complexity, DP is significantly easier than SNRS because DP can be implemented using pre-/post-processors to existing single-layer encoders, while SNRS cannot. In simulations, the physical-layer bit rate is held constant at $4.58 \mathrm{Mb} / \mathrm{s}$, and the bit rate for video is determined based on FEC overhead and, for the two-layer techniques, the percentage of data in each layer.

Figure 13 shows the effect of cell-level coding on PSNR for the public-domain Flower Garden video sequence encoded using a single-layer MPEG-2 encoder operating at a video bit rate of $4 \mathrm{Mb} / \mathrm{s}$. The curve labeled "No loss" corresponds to video performance when there are no packet losses for different cell coding parameters. The decrease in PSNR in this curve for large cell-level FEC represents the loss due to overhead bandwidth consumed by the redundancy in FEC codes. The delay spread values used for simulating various environments are indicated on the graph. A value of $88 \mathrm{~ns}$ corresponds to a large-size office, whereas 176 to $220 \mathrm{~ns}$ delay spread range corresponds to meeting rooms, classrooms, or auditoriums. We conclude from these results that cell-level coding with 10 to 20 redundant cells in a block of 256 cells results in acceptable performance for large-size offices. Large auditoriums require larger overheads, 50 cells in blocks of 256 cells. Currently, off-the-shelf R-S codecs with parameters $s=$ 10 exist for block sizes of 255 cells, whereas $s=50$ is an ambitious goal at this time.

Figure 14 compares the performance of the two-layer MPEG-2 codes with that of the single-layer code. The value of $s$ for the enhancement layer $\left(s_{e}\right)$ was set at 20 , and the best value of $s$ for the base layer was determined to be 70 for both DP and SNRS by means of experimentation. The results indicate that the single-layer algorithm can perform better in low error scenarios, but at larger delay spreads the two-layer scheme significantly outperforms the single-layer scheme. Furthermore, SNRS significantly outperforms DP throughout the range of delay spreads.

\section{Multi-Access Layer Issues for Wireless Packet (ATM) Networks}

$S$ ince ATM/B-ISDN connections may be stretched over wireless links in the future, the end-to-end performance of an ATM connection may be determined primarily by the performance of the wireless link(s). Two major issues are introduced into ATM technology development when the wireless aspect is added. These are:

- The shared use of unreliable transmission links

- Mobility of the terminals
Regarding the unreliability of transmission links, we have already addressed physical layer and data link layer issues in the fourth and fifth sections, respectively. In this section, we focus on the multi-access layer, which deals with the shared use of transmission links by multiple users. In the seventh section, we consider the final issue, mobility management.

\section{Types of Multi-Access Protocols}

Media access control or multi-access (MAC) protocols attempt to efficiently and equitably allocate use of a communications channel to independent, competing users. Errors result when two or more users simultaneously (in time and frequency) attempt to transmit over a channel. ${ }^{3}$

When such a conflict occurs, all involved packets must be retransmitted. A user hears if its transmission was successful one round-trip propagation time after transmitting a packet over the multiaccess channel. Propagation delays across the channel range from millisecond values for LANs to approximately $0.25 \mathrm{~s}$ in satellite applications.

Many channel access protocols have been proposed and analyzed in the past few decádes [20-23]. Each scheme has its advantages and limitations, providing acceptable performance only in certain environments and with certain types of channel traffic. Protocols are grouped into five classes [21]: fixed assignment, random access, centrally controlled demand assignment, demand assignment with distributed control, and adaptive strategies and mixed modes. In addition, three network environments are characterized [21]: satellite, ground radio, and local area. Each environment has its own unique characteristics, such as the magnitude of propagation delays. The performance of a protocol depends to a large extent on the environment.

Fixed-assignment techniques, such as TDMA and frequency division multiple access (FDMA), incorporate permanent subchannel assignments (in the time-frequency domain) for individual users. These "classical" schemes perform well with stream-type traffic (each user transmitting a steady flow of messages), such as voice. At all times, a large percentage of the subchannels carry user traffic. The result is high utilization of the communications channel, and low user response times. Fixed-assignment techniques, however, are inefficient in bursty traffic applications. A subchannel is wasted whenever its owner does not have anything to transmit.

Bursty traffic is serviced more efficiently by a random access protocol. The ALOHA and carrier sense multiple access (CSMA) schemes are typical examples. Random access techniques make the full channel capacity available to users, for short periods of time, on a random basis. They are packetoriented, whereas the fixed-assignment techniques are channel-oriented. They dynamically allocate transmission capacity on a per-packet basis.

The simplest random access protocol, pure ALOHA, permits users to transmit at will [24]. Whenever one user's transmission overlaps any part of another user's transmission, a collision occurs, and both messages must be retransmitted. When the channel is lightly loaded, few collisions occur in ALOHA-based schemes. Consequently, the expected delay, from arrival of a packet until its successful transmission, is very small. However, ALOHA schemes are inherently unstable.

Collision resolution algorithms [25] and group testing procedures [26] were invented to improve the maximum achievable stable throughput of random access protocols. Instead of

${ }^{3}$ In this section, we do not consider spread spectrum techniques (see the section on "Spread Spectrum"), such as CDMA, which allow multiple users to simultaneously transmit packets. 
retransmitting unsuccessful packets after random delays, distributed control algorithms schedule the retransmissions. Of primary importance, these techniques guarantee system stability, provided the input rate to the network is not too large.

For LANs, random access protocols take advantage of the short propagation delays between users. In CSMA, transmission is delayed until the channel is sensed idle. This reduces the number of collisions, and leads to high throughput and low delay [27]. If users also have the ability to detect collisions (e.g., CSMA with collision detection, CSMA/CD), additional performance gains result. Despite the improvements achieved with carrier sense techniques, stability problems persist. Furthermore, performance rapidly deteriorates as the maximum propagation delay between users increases. More important, it is very difficult to sense collisions in a wireless channel.

Like the random access protocols mentioned above, demand assignment techniques provide channel capacity to users on a demand basis, as they need it. Unlike random access, however, demand assignment involves two stages: a reservation stage followed by a transmission stage. Operating under random access protocols, users let the system know they have information to send simply by transmitting their data packets. Resulting collisions on the data channel involve the actual information-carrying packets.

Demand-assigned protocols, however, achieve high channel throughput by requiring users to reserve communications bandwidth. A portion of the channel capacity is required in this reservation stage. The reservation subchannel is accessed by users according to a multiple access protocol, typically TDMA or slotted ALOHA. Short reservation packets are sent to request channel time; the shorter they are, the less capacity necessary for the reservation subchannel. Once channel time is reserved, information packets are transmitted conflict-free. Conflicts occur only on the small-capacity reservation subchannel. At low throughputs, though, the message delay is increased over that of random access techniques. Users must wait for their reservations to be accepted, and for their assigned transmission times. Control of the reservation and transmission stages can be either centralized or distributed.

A common example of demand assignment with central control is polling: each user is addressed, sequentially by a central station, for transmission privileges. The proper operation of a centrally controlled system, however, depends on the reliability of the controller. Demand assignment with distributed control avoids this problem.

With distributed control, users base their actions entirely on information available to everyone. Broadcast channels provide full connectivity; hence, actions are determined by the transmission history of the channel. All users listen for reservation packets and apply the same distributed scheduling algorithm. Requests are made on either a contention or fixed-assignment basis.

The final class of multiple access techniques, the adaptive schemes and mixed modes, are designed to handle situations involving a combination of traffic types or a time-varying mixture. All protocols have their strengths and weaknesses; no access scheme outperforms all others under every condition. To accommodate a combination of traffic types, channels can be partitioned into several sections, each operating under its own protocol. Likewise, adaptive protocols attempt to provide good performance over a large range of conditions; the access scheme itself changes, adapting smoothly to network load fluctuations, yielding an access procedure appropriate for the actual state of the network.

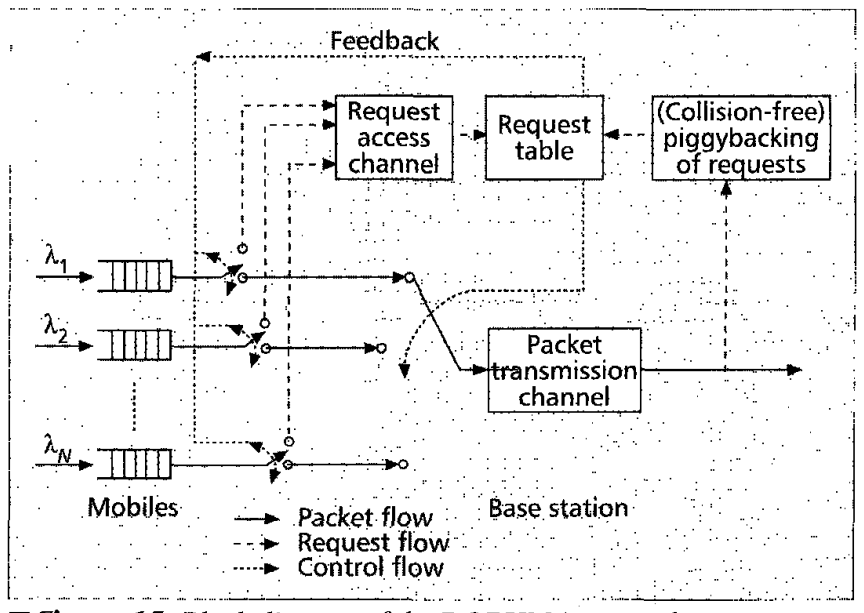

Figure 15. Block diagram of the DQRUMA protocol.

\section{An Example MAC Protocol: DQRUMA}

As noted above, many MAC protocols have been proposed and studied during the past few decades. The protocols allow shared, wireless access by multiple, mobile users. In a wireless packet (ATM) network that supports an integrated mix of multimedia traffic, the MAC protocol needs to be designed such that mobiles share the limited communications bandwidth in an efficient manner, maximizing the utilization of the frequency spectrum and minimizing the delay experienced by mobiles. Also, to provide acceptable end-to-end ATM performance, it is important to define an efficient MAC protocol that can satisfy QoS parameters such as cell delay variation and cell loss rate, and support various services such as constant, variable, available, and unspecified bit rate $(\mathrm{CBR}, \mathrm{VBR}$, ABR, and UBR).

In this section, we describe an efficient demand-assignment channel access protocol, which we call distributed-queuing request update multiple access (DQRUMA) [28]. We consider a cell (or microcell) in a wireless network with a base station and $N$ buffered mobiles. Fixed-length packets (or, e.g., ATM cells) arrive at the mobiles according to, in general, bursty random processes. The packets are buffered at the mobiles until they are transmitted "uplink" to the base station (according to the channel access protocol). The base station broadcasts "downlink" packets destined for mobiles within its cell. We assume the uplink (mobile-to-base) and downlink (base-to-mobile) communications are physically separate (e.g., on different frequency channels). There can be multiple uplink and downlink frequencies, but for simplicity herein we assume there is a single uplink and single downlink frequency.

Figure 15 shows a block diagram of the proposed DQRUMA protocol, and Fig. 16 shows a flow chart of the DQRUMA protocol at each mobile. We consider a time-slotted system in which a request-access (RA) channel and a packet transmission (Xmt) channel are formed on a slot-by-slot basis (Fig. 17). If desired, larger or smaller numbers of RA channels can be allocated than indicated in Fig. 17 (e.g., RA channels may occur in every other time slot). However, perhaps the best way to match the RA channel bandwidth with the actual requirements is to use the dynamic procedures outlined in [28].

As the base station receives transmission requests (by listening to the uplink RA channel) from the mobiles, the base station updates the appropriate entries in a request table. The request table contains an entry for all $N$ mobiles in the system; each entry in the table contains a mobile's short $b$-bit access identification (access ID) and an associated field containing transmit-request (Xmt_Req) information (e.g., whether or not the mobile has any more packets to transmit). 


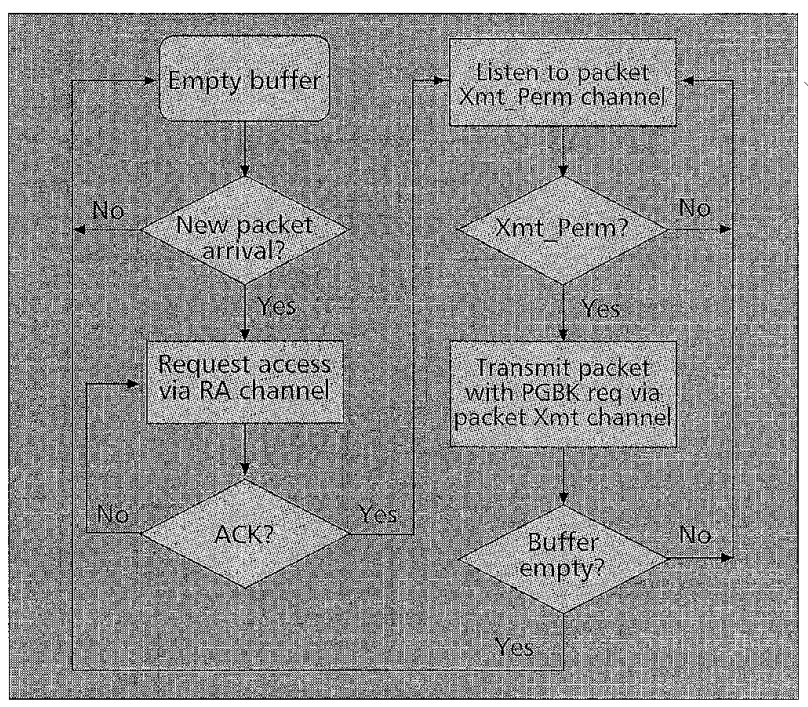

Digure 16. Flow chart of the DQRUMA protocol at each mobile.

When a packet (or packets) arrives to a mobile with its buffer empty, the mobile sends a Xrnt_Reo to the base station via the uplink RA channel - perhaps in contention with other mobiles according to some random access protocol. The Xnt_Req from a mobile includes the mobile's $b$-bit access ID, which was assigned at call setup or handoff. When the base station successfully receives a Xmt_Req from a mobile, it sets the corresponding entry in the request table to indicate that the mobile has a packet(s) to transmit (this is the "request" aspect of the DQRUMA protocol). The base station also acknowledges reception of the Xmt_Req by broadcasting the b-bit Access ID over the downlink ACK channel (Fig. 17). Here, we assume negligible propagation delays between the mobiles and the base station; generalization to larger propagation delays are considered in [28].

Once a mobile receives positive acknowledgment (by listening to the downlink ACK channel) that its Xmt_Req was received by the base station, it listens to the downlink transmit-permission (Xmt_Perm) channel every time slot until it hears its $b$-bit access ID, which signifies permission from the base station to transmit a packet during the next time slot. This is the "distributed queuing" aspect of the DQRUMA protocol: packets are queued at mobiles, and the base station "serves" them according to a desired packet transmission policy (e.g., round-robin). The transmit permission is given each time slot to one of the mobiles that has a nonempty Xmt_Req field in the request table.

Each time a mobile transmits a packet (using the uplink Xmt channel), it also includes a piggybacking (PGBK) request bit to indicate whether it has more packets in its buffer (Fig. 17). This piggybacking serves as a contention-free Xmt_Req for the mobile transmitting a packet; the base station checks the $\mathrm{PGBK}$ request bit and updates the appropriate entry in the request table accordingly (this is the "update" aspect of the DQRUMA protocol). Once a mobile successfully sends a Xmt Req over the RA channel and as long as its buffer remains nonempty, the Xmt_Regs for additional packets arriving at the mobile are sent to the base station by piggybacking the requests in a contention-free fashion. In other words, only packets arriving at a mobile's empty buffer will send a Ximt Req over the RA channel; consequently, contention for use of the RA channel is dramatically reduced and the overall protocol performance greatly improved.

Furthermore, mobiles that transmit packets of certain "service classes" (e.g., CBR traffic) do not need to place
Xmt_Regs. The base station can be programmed (e.g., at call setup) to automatically generate "implicit" Xmt_Reqs so that the corresponding entries in the request table are updated at the appropriate (periodic) time instants. This feature helps minimize the amount of traffic on the RA channel.

Thus far we have not mentioned anything about the particular algorithms to be used for the RA channel and the Xmt channel. Some examples are provided in [28], but the DQRUMA protocol presented so far is general in the sense that, according to different traffic and service requirements, any random access protocol can be used for the RA channel, and any scheduling policy can be used by the base station for granting mobiles permission to transmit on the uplink Xmt channel. The explicit slot-by-slot announcement of the packet Xmt_Perm allows the base station to implement, if desired, an "optimal" scheduling of packet transmissions for integrated mixes of traffic with multiple priority levels and service requirements.

\section{Mobility Management for Wireless Packet (ATM) Networks}

$T$ o handle the mobility of terminals in a wireless ATM network, there is a need to address the following three basic issues [40]:

- Location management

- Connection management

- Handoff management

In this section, we provide a high-level description of the algorithms to track and locate mobiles, admit connections, and perform handoffs.

Location management has two functions: tracking the position of a mobile (with registrations) and handling queries regarding the location of a mobile (for example, prior to call delivery to a mobile). The IS-41 standard for location management requires databases called home location register (HLR) and visitor location register (VLR). This may be a valid model to adopt for wide-area wireless ATM networks. However, for LANs other solutions based on broadcasting may be simpler to implement without requiring the overhead of maintaining these location servers. Such solutions were proposed, for example, as part of the BAHAMA project at Bell Laboratories (see the section on "Bell Laboratories"). Besides the LAN/WAN dimension, the algorithms and protocols defined for mobility management should also allow for the possibility of terminating the ATM protocol at the mobile or at the base station (see the third section).

ATM is a connection-oriented technology, with a connection establishment phase prior to data exchange and a connection release phase after data exchange. In mobile networks, as endpoints move, segments of connections need to be torn down and reestablished. Meanwhile, maintaining cell sequence and connection QoS while performing handoffs are important requirements in wireless ATM networks. Reference [29] classifies different connection rerouting methods for handoffs into three categories: anchor rerouting used in IS-41 and GSM standards-based cellular networks [30,31], dynamic rerouting [32], and pre-established tree routing [33]. The first two schemes require segment tear down and setup during handoffs. To minimize handoff time and packet loss during handoffs, schemes such as the preestablished virtual connection trees approach of [33] are being proposed for wireless ATM networks. The latter avoids segment teardown and setup during handoffs by setting up extra segments of a connection tree (from a root switch to a set of base stations to which the mobile could potentially move) during the connection setup phase. 
Connectionless networks completely avoid this problem of having to tear down and set up segments or establish extra segments for handoffs. Since routing in connectionless networks is based on destination addresses, a simple update of the current location of the destination is sufficient to handle mobility. Typically, first, a forwarding pointer is stored in the old router to point to the new router/base station through which the mobile endpoint can be reached. Subsequently, the far-end host or router updates its routing data so that datagram packets are delivered directly to the new router/base station. Such schemes have been developed for Mobile IP in [34].

In Mobile IP, if an end host moves, packets destined to this host will be routed to the old router and then forwarded for some period of time before the far-end routers are updated with the mobile's current location. On the other hand, packets sent by the mobile continue to be sent directly to the far-end host. Since these packets may follow a different route from those sent by the mobile from its prior location, it is likely that packets will arrive out of sequence at the far-end host. This does not pose a problem because IP is not designed to maintain packet sequence.

To simplify handoffs in the BAHAMA project at Bell Laboratories, we emulate connectionless networks using ATM technology [17]. Instead of using the common interpretation of the VPI field, VPIs are assigned as addresses of network nodes (switches). All cells with a given VPI are routed to the same destination switch. This is accomplished by pre-establishing VPI sink (destination-rooted) trees. Individual connections within a VPI tree are distinguished using VCIs. This type of networking emulates "connectionless" data transfer in that packets are routed as per the destination address in the packet (cell) headers.

Given this form of "connectionless" networking in BAHAMA, we proposed the homing algorithm for handoffs [17]. In a mobile environment, handoff is a challenging problem, particularly when the traffic is packet-switched and we need to deliver the packets (ATM cells) to the mobiles in their proper first-in first-out (FIFO) sequence.

In the Homing algorithm, we introduce the concept of source home station and destination home station. These refer to particular base stations, associated with a connection, which play a pivotal role in maintaining cell sequence. ATM cells from user A destined to user B are first routed from A to the home station for $A$. The cells are then routed along a predetermined virtual path from the source home station to the destination home station, where they are buffered and then delivered in-sequence to B's local station. The term local station refers to the base station associated with a mobile's current position. To avoid inefficiencies in routing, the local stations of users are "slowly" updated to be their home stations.

The advantages of this homing algorithm include simple control and preservation of FIFO cell sequence within a connection. The implementation allows the preservation of FIFO cell sequence without a centralized controller and without resequencing at the destination. Key parts of the algorithm include:

- Delaying cells arriving at the home station from the new local station until all cells arrive from the old local station (reception at the home station of a special "tail" signal indicates that the path from the previous local station is clear).

- Retransmitting the appropriate cells from the home station as the handoff occurs from one base station to the next. Sequence numbers can be used between the destination

home and the mobile user, so the mobile can tell the home the number of the last cell it received before moving, and the destination home can retransmit cells, if necessary.

The homing algorithm differs from the anchor rerouting handoff schemes and the dynamic rerouting handoff scheme in that no connection segment teardown and setup occurs during handoffs. Nor is there any extra segment setup during connection establishment prior to data exchange, as is done in the pre-established tree routing handoff scheme. Instead, VPI trees are provisioned and maintained by a management-plane algorithm requiring no segment setup during handoffs or prior to data exchange. Thus, the homing algorithm is different from the handoff approaches used in connection-oriented networks.

Comparing the homing algorithm with handoff schemes used in connectionless mobile networks, such as Mobile IP, we observe that the homing algorithm needs to be more sophisticated than Mobile IP since it has to meet an important sequenced delivery requirement. A second important difference is that, unlike IP, the BAHAMA ATM network provides QoS guarantees.

\section{Interconnection and Interoperability with Other Networks}

$f$ the wireless ATM system is designed so that the wired part of the network strictly conforms to ATM standards, interconnection of the network to an ATM network is straightforward. In some cases (e.g., in the network in [17]), the cell structure in the wireless part of the network is slightly different than a regular ATM network, in that the VPI/VCI field is utilized in a different fashion. In [17], the difference is small and a simple translation is sufficient to interconnect the wireless ATM network to a regular ATM network.

\section{Wireless ATM Proposals in the Literature}

$\mathrm{n}$ this section, we discuss some of the wireless ATM proposals in the literature. This list comprises both wireless LAN and residential broadband wireless access applications. Although it covers a wide range of wireless ATM proposals, this is not an exhaustive list of all wireless ATM proposals in the literature. 

nection. These VCIs change only at the terminal leaves of the network, whereas at the intermedi-

\section{NEC C\&C Research Laboratories}

The system described in [35] is an ATM network for personal communication networks. This paper proposes that ATM cell relay technology be the basis of the next-generation wireless transport architectures. This proposal is based on observations that the wireline networks are evolving towards integrated broadband communications with an ATM infrastructure. Although [35] recognizes that the duplication of all the wireline capabilities over the wireless medium may be difficult, it suggests that we "aim at system designs which provide qualitatively similar attributes even if quantitative equivalence with the fiber network may not be feasible," with the general goal "to design integrated services wireless networks that provide tetherless extensions of fiber-optic based ATM network capabilities in a relatively transparent, seamless, and efficient manner." It is considered that a bit rate of $8 \mathrm{Mb} / \mathrm{s}$ can be readily achieved in picocellular environments (radius $100 \mathrm{~m}$ ) and is feasible by using antenna and modulation enhancements in microcellular environments (radius $500 \mathrm{~m}$ ). The paper suggests the transmission of ATM cells in packets consisting of full or submultiple ATM cells over the air. The protocol stack for the wireless ATM part incorporates a wireless data link layer and a multi-access layer. The data link layer is based on error detection and retransmissions. The paper proposes and presents simulation results for a multi-access layer based on a dynamic reservation protocol. This protocol incorporates a QoS control method based on time-of-expiry-based scheduling policy. Handoffs are handled in the data link layer.

\section{Cambridge-Olivetti Research Laboratories}

An experimental wireless ATM LAN was built at CambridgeOlivetti Research Laboratories in 1994 [36]. This network operates at $10 \mathrm{Mb} / \mathrm{s}$, uses a QPSK radio at $2.45 \mathrm{GHz}(10 \mathrm{MHz}$ bandwidth available for short-range data links in buildings in the United Kingdom) and low-end ATM switches operating at $100 \mathrm{Mb} / \mathrm{s}$, and has a picocellular structure without frequency reuse. A picocell has a radius of about $10 \mathrm{~m}$. The multi-access layer employs slotted ALOHA with exponential backoff, implemented using Xilinx reprogrammable gate arrays; the slot size is equal to an ATM cell. Packets over the air are one ATM cell long; headers are appropriately altered to accommodate QoS, with the VPI/VCI field condensed. Errors are detected using a CRC field of 16 bits, and retransmissions (up to 10$)$ are used for error correction.

\section{Columbia University/IBM}

In a paper that deals with topology issues in a cellular radio access network with ATM transport technology, Acampora and Naghshineh proposed a structure called the virtual tree architecture [33]. They showed that this approach can support a very high rate of handoffs between cells. They state that this capability in turn enables very small size cells, and therefore very high capacity. The virtual tree architecture consists of ATM switches and cellular base stations connected to some of the ATM switches. The switches are connected via some physical network topology, on top of which is a virtual tree. The root of the tree is a fixed ATM switch node, and the leaves are the ATM switches with base stations. When a mobile is admitted to the network with a connection, it is given two VCIs, one in each direction. These VCIs can go through for that particular connection continue to be used. The basic idea in this system is that in this way the call processor is not involved in the handover, or the handover is accomplished in a distributed manner. The mobile can go through the boundaries of a given connection tree, and then the call processors carry out a handoyer. However, this is minimized by keeping the geographical coverage of a virtual connection tree large. The paper presents a timing scenario with a polling-based multi-access protocol.

\section{Bell Laboratories}

In [17], a wireless ATM LAN system called BAHAMA is proposed. The basic characteristic of the network is its ad hoc nature: the network is self-organizing; that is, a predetermined topology does not exist - the network elements, called portable base stations (PBSs), communicate to determine the network topology after changes due to the addition or deletion of network elements. PBSs are designed with simplicity in mind; therefore, the ATM segmentation and reassembly is carried out in the portable units. In order to support mobility in the simplest possible way, a new VPI/VCI concept is defined which supports routing based on the destination address. Mobility is supported by means of an adaptive homing algorithm. The network employs a wireless data link layer that provides high reliability, based on both ARQ and FEC. Multiple access is provided by DQRUMA. Currently, a project based on BAHAMA, named Mobile Information Infrastructure, is being carried out by Bell Laboratories and Sun Microsystems, and is supported by the U.S. National Institute of Standards and Technology.

\section{NTT AWA}

A system suggestion from NTT Wireless Systems Laboratories called $A W A$ (ATM Wireless Access) concentrates on wireless access to an ATM network at the super high frequency (SHF) band for private LAN/WAN applications as well as public environments. Propagation properties of the SHF band (3-30 $\mathrm{GHz}$ ) provide limitations on the system range. This proposal aims to provide higher-speed transmission than the Future Public Land Mobile Telecommunication Systems (FPLMTS) being standardized at the ITU (230 MHz has been assigned in the $2 \mathrm{GHz}$ band for speeds of $2 \mathrm{Mb} / \mathrm{s}$ ), or the Mobile Broadband Systems project conducted in the RACE program in Europe. The system is designed for ATM transport capability and is connected to ATM-based broadband networks for seamless ATM end-to-end connection. Since it is an access system, the system is designed for low-mobility applications. The $>10 \mathrm{GHz}$ SMF band suffers from more serious shadowing effects than the lower-frequency bands, but since lowerfrequency bands are not available in Japan, the system is being designed for this band. With a highly directive antenna, shadowing effects are lessened, and the frequency band being considered may make it possible to have small antennas. The system is designed as a dual wireless LAN and access to a ATM-based public multimedia network. A low degree of mobility will be supported. As in several other proposals, there are a specific link layer and multi-access layer designed for the wireless channel. In AWA, these layers are under the ATM adaptation layer, and replace part of the ATM layer. The specific way a cell is transmitted over the air is not defined, except that HEC is not present, and both a header 
and a trailer are considered possible, and both FEC and CRC are under consideration. Furthermore, wireless headers are treated separately than payloads in terms of error detection, and cells with errors at the header are discarded but those with errors in the payload are not, which is a surprising decision. Although this is in line with the basic ATM philosophy, ATM does not have error detection over the payload and typically operates over highly reliable media. A multiservice dynamic reservation TDMA approach is chosen for multi-access purposes. Time stamps (of length 2 bytes/ATM cell) are added to each ATM cell header to control QoS, which is restricted to cell delay variation. After a study of the required transmission rates for various applications, the authors believe that $10 \mathrm{Mb} / \mathrm{s}$ is sufficient to provide high-quality services for personal communications. Since this is the approximate target rate per user in the AWA system, an air interface rate of $30-80 \mathrm{Mb} / \mathrm{s}$ one way is needed (they employ TDD and FEC). For modulation, the authors suggest QPSK and $\pi / 4-$ QPSK with differential detection. According to their system studies, the authors believe that this system can be built with a micro- or picocellular architecture.

\section{Carleton University}

In a project sponsored by the Canadian Institute for Telecommunications Research (CITR), researchers at Carleton University led a collaborative effort carried out by researchers from three research institutions and seven universities [38]. This is a high-speed $(160 \mathrm{Mb} / \mathrm{s})$ access system using a radio within the 20 to $60 \mathrm{GHz}$ range. Leading-edge millimeter-wave and surface-acoustic-wave (SAW) technologies are employed to design the implementation of the system. Switched multibeam sectored antennas are employed at the base station and the mobile. With the use of TDM, the net maximum offered bidirectional traffic per microcell will be $60-75 \mathrm{Mb} / \mathrm{s}$, while allowing different uplink and downlink rates within the 60-75 $\mathrm{Mb} / \mathrm{s}$ bidirectional rate. The system will have a cellular structure with cells of radii about $30-50 \mathrm{~m}$. An anti-multipath measure based on time diversity is employed. The multi-access protocol is based on an adaptive-rate polling scheme, where ATM cells are packaged in 64-byte envelopes. The error control system is based on ARQ, supplemented by sector or base station handoff to combat "slow" effects of fading. A demonstration system operating at $40 \mathrm{Mb} / \mathrm{s}$ is being developed in 1996 incorporating monolithic microwave integrated circuit (MMIC)/miniature hybric MIC (MHMIC) modules, microstrip antennas, and SAW-based anti-multipath measures.

\section{ACTS Magic WAND}

Magic WAND (Wireless ATM Network Demonstration) is a joint European project to develop a demonstration of mobile terminals for multimedia information access using a fast and wireless ATM network [39]. The project is sponsored by the European Council Advanced Communications Technologies and Services (ACTS) program. Magic WAND is a three-year project which started in October 1995. The project has six companies and five universities as project partners. The project focuses on a $5 \mathrm{GHz}(20 \mathrm{Mb} / \mathrm{s})$ demonstration system and does research on $17 \mathrm{GHz}$ tachnology. The overall goal is to design a wireless ATM access network demonstration system that can be commercialized and standardized in ETSI. It is expected that Magic WAND will support location-independent, medium-capacity $(20 \mathrm{Mb} / \mathrm{s})$ access to broadband infrastructure networks. The project will address general system design, radio physical layer, MAC, signaling, control, and network management, system integration and evolution, user trials, and standardization issues.
M/ ith the increasing popularity and power table computers and wireless $L A N$ applications, and ess applications will become highly desirable.

\section{Summary and Conclusions}

$A$ TM technology for the transport of broadband integrated communications is becoming ubiquitous, especially in LANs and residential video delivery. High-speed ATM switching fabrics will soon be available in increasing capacities and at decreasing prices. With the increasing popularity and power of portable computers and wireless LAN applications, and the increasing need for ATM to the home, ATM-based wireless access applications will become highly desirable. Technology is becoming accessible to provide wireless ATM systems. The most important issues in the design of wireless LANs are in the physical layer, data link layer, multi-access control, and mobility management. In this article we provided a survey of the key issues in each of these topics and gave examples of problem solutions. From the list of several ongoing design and prototyping projects in the United States, Canada, Japan, and Europe, and from the recent increased activity on wireless ATM in the ATM Forum, it is clear that commercially available wireless ATM systems are in the not too distant future.

\section{Acknowledgments}

The authors would like to thank Sirikiat Ariyavisitakul, Len Cimini, Babak Daneshrad, Pramod Pancha, Amy Reibman, and Malathi Veeraraghavan for many discussions, and Joseph Nordgaard for supplying Figs. 4 and 5.

\section{References}

[1] J. P. Coudreuse, P. Adam, and P. Gonet, "Asynchronous Time-Division Switching: The Way to Flexible Broadband Communication Networks," Proc. Int'l. Zurich Seminar on Digital Commun. '86, Mar. 1986.

[2] E. Geiger et al., "Network Compatible ATM for Local Network Applications, Phase 1," version 1.01, Oct. 1992. Available at $\mathrm{ftp}: / / \mathrm{ftp}$.bellcore.$\mathrm{com} / \mathrm{pub} / \mathrm{nclatm} / \mathrm{nclatm}-1.01 . \mathrm{ps}$

[3] T. Kwok, "A Vision for Residential Broadband Services: ATM-to-theHome," IEEE Network, vol. 9, Sept. 1995, pp. 14-28.

[4] D. F. Bantz and F. J. Bauchot, "Wireless LAN Design Alternatives," IEEE Network, vol. 8, Mar. 1994, pp. 43-53.

[5] J. E. Padgett, C. G. Gunther, and T. Hattori, "Overview of Wireless Personal Communications," IEEE Commun. Mag., vol. 33, Jan. 1995, pp. 28-41.

[6] K. Pahlavan and A. H. Levesque, "Wireless Data Communications," Proc. IEEE, vol. 82, Sept. 1994, pp. 1398-1430.

[7] Kluwer Wireless Personal Communication Journal, Special Issue on Multi-Carrier Modulation, Jan. 1996.

[8] G. McGibneyet al., "Implementation of a High-Performance Wireless LAN," Proc. IEEE ICUPC '94, San Diego, CA, Oct. 1994.

[9] A. S. Acampora and Z. S. Zhang, "A Throughput Delay Comparison: Narrowband Versus Broadband Wireless LANs," IEEE Trans. Vehicular Tech vol. 42, Mar. 1993, pp. 266-73.

[10] P. Nobles and F. Hatsall, "OFDM for High Bit Rate Data Transmission Over Measured Indoor Radio Channels," Proc. IEE Colloquium on Radio LANs and MANs, London, U.K., Apr. 1995.

[11] E. Biglieri et al, Introduction to Trellis-Coded Modulation with Applications, New York: Macmillan, 1991.

[12] J. Proakis, "Adaptive Equalization for TDMA Digital Mobile Radio," IEEE Trans. Commun., vol. COM-40, May 1991, pp. 333-41.

[13] E. Ayanoglu et al., "Forward Error Control for MPEG-2 Video Transport in a Wireless ATM LAN," to be published, ACM/Baltzer Wireless Networks $J$.

[14] J. H. Winters, J. Salz, and R. D. Gitlin, "The Impact of Antenna Diversity on the Capacity of Wireless Communication Systems," IEEE Trans. Commun., vol. 42, Feb. 1994, pp. 1740-51.

[15] G. Foschini and M. Gans, "On Limits of Wireless Communication in a Fading Environment when using Multiple Antennas," submitted for publication.

[16] E. Ayanogłu et al., "AIRMAIL: A Link-Layer Protocol for Wireless Networks," ACM/Baltzer Wireless Networks J., vol. 1, Issue 1, 1995, pp. 47-60. 


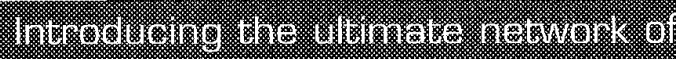

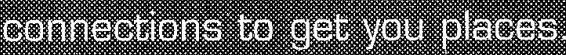

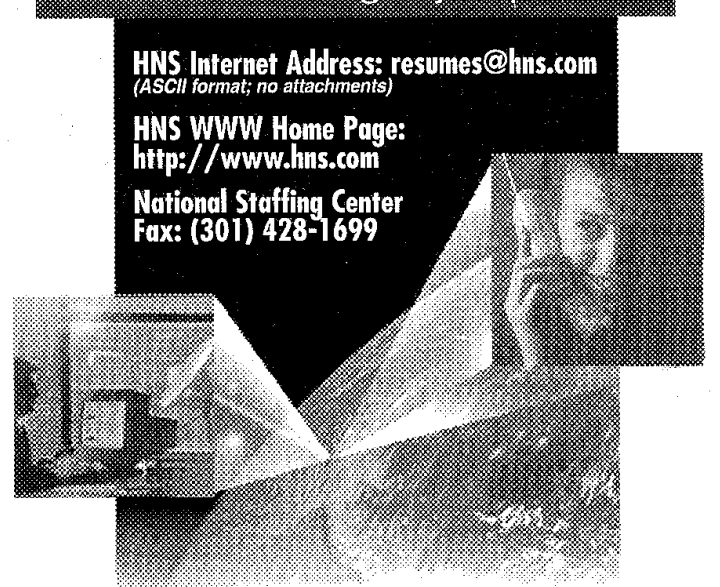

One of the world's fastest-

growing telecommunications companies, Hughes Network

Systems (HNS) provides wide-area network solutions that set the pace in the industry. With our unique combination of wireline and wireless technologies including satellite, cellular communications, PCS and enterprise network switching - we've grown at a compounded rate of nearly $40 \%$ over the last seven years. And the opportunities for future growth in the international, commercial market are unlimited. Connect with HNS - multiple positions exist in the following areas for qualified candidates:

\section{Sales \& Marketing}

Managers

Systems Engineers

Test Engineers

Field Engineers -

(Domestic/International)

Marketing Managers

Software Engineers

Hardware Engineers

Senior Product Engineers

Product Line Managers

CDPD Product Line

Managers

Product Qualification

Engineers (all levels)

Software Engineering

Manager (ICO)

Audio/Acoustics

Engineers

Firmware Engineers

Applications/Product/

Project Engineers

Digital Design Engineers

Real-Time Operating

System

RF Designers

MIS Engineers

Wide area networks: Wide open opportunities.

connections listed above to forward your resume (ASCll format; no attachments) or find out more about HNS.

Or mail your resume, indicating location preference, to: Hughes Network

Systems, Dept ATRADE

11717 Exploration Lane, Germantown, MD 20876; or San Diego: Hughes Network Systems, Dept. ATRADE, 10450 Pacific Center Court San Diego, CA 92121. An equal opportunity employer.
[17] K. Y. Eng et al., "A Wireless Broadband Ad-Hoc ATM Local-Area Network," ACM/Baltzer Wireless Networks J., vol. 1, 1ssue 2, 1995, pp. 161-74.

[18] E. Ayanoglu et al., "Forward Error Control for MPEG-2 Video Transport in a Wireless ATM LAN," to be published, Proc. IEEE ICIP '96.

[19] W. C. Jakes, Edd. Microwave Mobile Communications, Wiley, 1974 (Reissued by IEEE Press, 1994)

[20] L. Kleinrock, Queuing Systems, Volume 2: Computer Applications, New York: Wiley-Interscience, 1976

[21] F. A. Tobagi, "Multiaccess Link Control," Computer Network Architectures and Protocols. P. E. Green, Jr. ed. New York: Plenum Press, 1982.

[22] S. S. Lam, "Multiple Access Protocols," Computer Communications, Volume 1: Principles, Englewood Cliffs, NJ: Prentice Hall, 1983.

[23] R. G. Gailager, "A Perspective on Multiaccess Channels," IEEE Trans. info. Theory, vol. IT-31, Mar: 1985, pp. 124-42

[24] N. Abramson, "The ALOHA System: Another Alternative for Computer Communications," AFIPS Conf. Proc, vol. 37, Montvale, NJ: AFIPS Press, 1970, pp. 281-85.

[25] J. L. Massey, "Collision-Resolution Algorithms and Random-Access Communications" Multi-User Communication Systems (CISM Courses and Lectures Series, No. 265), Springer-Verlag, 1981.

[26] T. Berger et al., "Random Multiple-Access Communication and Group Testingr" IEEE Trans. Commun., vol. COM-32, July 1984, pp. 769-79.

[27] L. Kleinrock and F. A. Tobagi, "Packet Switching in Radio Channels Part 1: Carrier Sense Multiple Access Modes and Their ThroughputDelay Characteristics," IEEE Trans. Commun., vol. COM-23, Dec. 1975 pp. 1400-16

[28] M. J. Karol, Z. Liu, and K. Y. Eng, “Distributed-Queuing Request Update Multiple Access (DQRUMA) for Wireless Packet (ATM) Networks," Proc. ICC'95. June 1995, pp. 1224-31.

[29] T-H Wu and L. F. Chang, "Architectures for PCS Mobility Management on ATM Transport Networks," Proc. ICUPC '95, Nov. 1995, pp. 763-68.

[30] EIATIA IS-41 (Revision B), "Cellular Radio-telecommunications Intersystem Operations," Jan. 1992

[31] GSM Rec 0.9.02 "Mobile Application Part (MAP) Specification."

[32] L. V. Hauwermelren et al., "Requirements for Mobility Support in ATM," Proc, Globecom '94, pp. 1691-95, Nov. 1994.

[33] A. S. Acampora and M. Naghshineh, "An Architecture and Methodology for Mobile-Executed Handoff in Cellular ATM Networks," IEEE ISAC, vol. 12. Oct 1994 pp. 1365-75.

[34] C. E. Perkins and P. Bhagwat, "A Mobile Networking System Based on internet Protocol," IEEE Pers. Commun., 1st qtr, 1994, pp. 32-41.

[35] D. Raychaudhuri and N. D. Wilson, "ATM-Based Transport Architecture for Multiservices Wireless Personal Communication Networks" IEEE JSAC, vol. 12 Oct. 1992, pp. 1401-14.

[36] J. Porter and A. Hopper "An ATM-Based Protocol for Wireless LANs," Olivetti Research Ltd. Tech. Rep. 94.2, Apr. 1994. Available at ftp:// ftp.cam-orl.co.uk/pub/docs/ORL/tr.94.2.ps.Z

[37] M. Umehira et al., "An ATM Wireless Access System for Tetherless Multimedia Services " Proc. ICUPC '95. Tokyo, Japan, Nov. 1995.

[38] D. D. Falconer, "A System Architecture for Broadband Millimeter Wave Access to an ATM LAN," Proc. Wireless '95, Calgary, Canada, July 1995.

[39] The Magic WAND Wireless ATM Demonstrator is available at http://www.tik.ee.ethz.ch/ - wand.

[40] M. Veeraraghavan, M. Karol, and K. Y. Eng, "Mobility and Connection Management in a Wireless ATM LAN," to appear in IEEE JSAC, special issue on wireless ATM.

\section{Biographies}

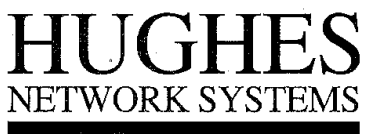

A HUGHES ELECTRONICS COMPANY

ENDER AYANOGLU [SM] received the B.S. degree from the Middle East Technical University in 1980 and the M.S. and Ph.D. degrees from Stanford University in 1982 and in 1986, all in electrical engineering. Since then, he has been with Bell Laboratories. During 1990-1991 and in 1992 he taught at Bilkent University, on leave from Bell Laboratories. His current research interests are in wireless ATM; particularly physical-layer and data-link-layer issues, multiwavelength optical networking; and general source and channel coding applications in communication networks.

KAI Y. ENG [F] received his B.S.E.E. (summa cum laude) from Newark College of Engineering, New Jersey, in 1974, and his M.S. and Dr.Engr.Sc. degrees in electrical engineering from Columbia University, New York, in 1976 and 1979, respectively. From 1974 to 1979, Dr. Eng was with RCA Astro-Elec tronics (Princeton, New Jersey). where he worked on communications satellites and shaped-beam antenna designs. Since 1979, he has been with Bell Laboratories (Holmdel, New Jersey), where he is currently the head of the Broadband Systems Research Department.

MARK J. KAROL [F] received the B.S. degree in mathematics and the B.S.E.F. degree in 1981 from Case Western Reserve University, and the M.S.E. M.A., and Ph.D. degrees in electrical engineering from Princeton University in 1982, 1984, and 1986, respectively. Since 1985 he has been a member of the Research Communications Sciences Division at Bell Laboratories (formerly part of AT\&T, now part of Lucent Technologies) in Holmdel, New Jersey. He is currently a Distinguished Member of Technical Staff. 